\title{
THE TOPOLOGICAL DEGREE METHOD FOR EQUATIONS OF THE NAVIER-STOKES TYPE
}

\author{
V. T. DMITRIENKO AND V. G. ZVYAGIN
}

\begin{abstract}
We obtain results of existence of weak solutions in the Hopf sense of the initial-boundary value problem for the generalized Navier-Stokes equations containing perturbations of retarded type. The degree theory for maps $A-g$, where $A$ is invertible and $g$ is $\mathcal{A}$-condensing, is used.
\end{abstract}

Various problems for the Navier-Stokes equations describing the motion of the Newton fluid, and its generalizations for nonlinearly-viscous and viscoelastic fluids, have been developed in many papers. We mention here some of the papers which contain surveys on this subject, different approaches, constructions, and methods of investigation: [1], [8], [10]-[16].

Here we consider the problem of the existence of weak solutions, in the Hopf sense, of the initial-boundary value problem for equations of the NavierStokes type. These equations include the ones describing the movement of nonlinear-viscous and viscous-elastic fluids. We reduce the above problem to an evolution equation in the space of functionals, and then to the equivalent operator equation. The method of this paper consists of constructing operator equations which approximate the original ones, and then investigating their solvability by means of infinite-dimensional degree theory. As we know, the Galerkin-Faedo method or iteration methods have already been used instead of the degree theory for the classical Navier-Stokes equations and for some their generalizations (see, for example, [1], [10], [12]-[15]). The solution of the original problem may be obtained by passage to the limit in the set of solutions of approximating equations. The results of our paper on the existence of weak solutions generalize the well known ones (see, for example, [2], [10], [13], [15]).

1991 Mathematics Subject Classification. Primary 47H17.

Key words and phrases. Weak solutions, Navier-Stokes equations, a priori estimates, degree theory, $\mathcal{A}$-condensing perturbations.

Received: March 26, 1997. 
This paper consists of four sections.

In the first section we introduce the main notations and notions, set up the problem of weak solutions of the initial-boundary value problem for generalized Navier-Stokes equations, and formulate our main results of existence and uniqueness of weak solutions.

In the second section the problem of weak solutions is reduced to the investigation of an equivalent operator equation. Then we construct the approximating equations and investigate the properties of the operators involved.

In the third section a priori estimates of solutions of approximating equations are established and a proposition on the existence of solutions of such equations is obtained.

In the last section the possibility of the limit procedure in the sequence of solutions of approximating equations is established. We present two different approaches to proven the convergence and, as a corollary, we get propositions for the existence of weak solutions of the initial-boundary value problem for some cases of the generalized Navier-Stokes equations. We consider the uniqueness of solutions for dimension $n=2$ as well.

It should be noted that our interest in this problem arose when Professor P. E. Sobolevskii posed to one of the authors the question of the applicability of topological methods to the initial-boundary value problems in hydrodynamics. The authors are grateful to P. E. Sobolevskii, and Yu. A. Agranovich for discussions on some problems in hydrodynamics.

\section{Introduction. Statement of the Problem. Main Results}

1.1. Notations. Let $\Omega$ be a bounded domain in $\mathbb{R}^{n}$ with the boundary $\partial \Omega$ of class $C^{2}$. For $T>0$, we denote by $Q_{T}$ the cylinder $(0, T) \times \Omega$. The bar over $\Omega, Q_{T}$ means closure.

We consider different spaces of functions on $\Omega$ with values in $\mathbb{R}^{n}$ :

$L^{2}(\Omega)$ denotes the space of square integrable functions on $\Omega$. The scalar product of functions $u$ and $v$ from $L^{2}(\Omega)$ is defined by $(u, v)=$ $\int_{\Omega} u(x) \cdot v(x) d x$; the norm of the function $u$ in $L^{2}(\Omega)$ will be denoted by $\|u\|_{L^{2}(\Omega)}$;

$W_{2}^{1}(\Omega)$ denotes the space of functions which belong together with their first order partial derivatives to $L^{2}(\Omega)$. A norm of the function $v$ from $W_{2}^{1}(\Omega)$ is defined by the following equality

$$
\|v\|_{W_{2}^{1}(\Omega)}=\left(\sum_{i=1}^{n}\left\|\frac{\partial v}{\partial x_{i}}\right\|_{L^{2}(\Omega)}^{2}+\|v\|_{L^{2}(\Omega)}^{2}\right)^{\frac{1}{2}} ;
$$

$\mathcal{D}(\Omega)$ denotes the space of functions of class $C^{\infty}$ with a compact support in $\Omega$.

$W_{2}^{1}(\Omega)$ denotes the closure of the set $\mathcal{D}(\Omega)$ with respect to the norm of the space $W_{2}^{1}(\Omega)$.

Denote by 
$\mathcal{V}=\{v \in \mathcal{D}(\Omega): \operatorname{div} v=0\}$ the set of solenoidal functions;

$H$ the closure of $\mathcal{V}$ with respect to the norm of the space $L^{2}(\Omega)$;

$V$ the closure of $\mathcal{V}$ with respect to the norm of the space $W_{2}^{1}(\Omega)$.

Norms and scalar products in the spaces $H$ and $V$ are defined by the same way as in spaces $L^{2}(\Omega)$ and $W_{2}^{1}(\Omega)$ respectively.

Also in the space $V$ the symbol of another scalar product will be used $((u, v))=\sum_{i=1}^{n}\left(\frac{\partial u}{\partial x_{i}}, \frac{\partial v}{\partial x_{i}}\right)$. And the norm generated by this scalar product in the space $V$ is equivalent to the norm induced from the space $W_{2}^{1}(\Omega)$.

Let $V^{*}$ denote the dual space to $V$, and $\langle h, v\rangle$ means action of the functional $h$ from $V^{*}$ to the element $v$ from $V$.

Also we consider spaces of functions $v:[a, b] \rightarrow X$ with values in a Banach space $X$. In what follows,

$L^{\alpha}((a, b), X)$ denotes the space of functions which are integrable with the power $\alpha \geq 1$. The norm of a function $v$ from $L^{\alpha}((a, b), X)$ is defined by the equality

$$
\|v\|_{L^{\alpha}((a, b), X)}=\left(\int_{a}^{b}\|v(t)\|_{X}^{\alpha} d t\right)^{1 / \alpha} .
$$

$L^{\infty}((a, b), X)$ denotes the space of essentially bounded functions with the norm

$$
\|v\|_{L^{\infty}((a, b), X)}=\operatorname{vrai} \sup _{t \in(a, b)}\|v(t)\|_{X} ;
$$

$C([a, b], X)$ denotes the space of continuous functions with the norm

$$
\|v\|_{C([a, b], X)}=\max _{[a, b]}\|v(t)\|_{X} .
$$

The spaces described above are Banach ones. In the case, when the interval $[a, b]$ is clear from a context, the notation $[a, b]$ is omitted: $L^{\alpha}(X), L^{\alpha}(X)$, $C(X)$. A dual space for a space $L^{\alpha}((a, b), X)$ is the space $L^{\alpha^{\prime}}\left((a, b), X^{*}\right)$, where $\frac{1}{\alpha}+\frac{1}{\alpha^{\prime}}=1$.

For vector-function $v$ from $L^{\alpha}((0, T), V)$ we denote:

by $v_{i}$ the coordinate functions;

by $\frac{\partial v}{\partial x_{i}}, \frac{\partial v}{\partial t}$ the first order partial derivatives;

by $D^{1} v=\left(\frac{\partial v_{i}}{\partial x_{j}}\right)$.

Let us introduce the following notations. Let

$X=L^{2}((0, T), V)$ with the norm $\|v\|_{X}=\|v\|_{L^{2}((0, T), V)}$ for $v \in X$,

$X^{*}=L^{2}\left((0, T), V^{*}\right)$ with the norm $\|f\|_{X^{*}}=\|f\|_{L^{2}\left((0, T), V^{*}\right)}$ for $f \in X^{*}$,

$W=\left\{v ; v \in X, v^{\prime} \in X^{*}\right\}$ with the norm $\|v\|_{W}=\|v\|_{X}+\left\|v^{\prime}\right\|_{X^{*}}$. 
1.2. The statement of the problem. The equations with perturbations of retarded type arise in mechanics for visco-elastic materials. By the definition (see [5]), "these materials are such that they have "memory" in sense that at the moment $t$ the tension state depends on all the deformations to which the material have been undergone".

If we reject the proportional dependence

$$
D=\mu \mathcal{E}
$$

between the stress tensor $D$ and the strain velocity tensor $\mathcal{E}$ we obtain the non-Newton or real fluids.

We would like to point out some mathematical models describing motion of such fluids.

In the paper [13] Litvinov V.G. investigated equations of fluid motion with relations

$$
\begin{aligned}
& D=\varphi\left(I_{2}\right) \mathcal{E}, \quad \mathcal{E}=\left(\varepsilon_{i j}\right), \quad I_{2}^{2}=\sum_{i, j=1}^{n} \varepsilon_{i j}^{2}, \\
& D=\varphi_{1}\left(I_{2}\right) \mathcal{E}+\varphi_{2}\left(I_{2}\right) \mathcal{E}^{2} .
\end{aligned}
$$

The Oldroid relation

$$
\lambda_{1} \frac{d D}{d t}+D=\nu_{0}\left(\mathcal{E}+\chi_{1} \frac{d \mathcal{E}}{d t}\right)
$$

leads to investigation of fluids with "memory". Solving the equation concerning $D$ we obtain

$$
D=\nu_{0} \mathcal{E}+\frac{\chi_{1}-\lambda_{1}}{\nu_{0}} \int_{0}^{t} e^{-\frac{t-s}{\lambda_{1}}} \mathcal{E} d s
$$

Substituting the expression for $D$ into the Cauchy form of the motion equation

$$
\rho\left(\frac{\partial v}{\partial t}+\sum_{i=1}^{n} v_{i} \frac{\partial v}{\partial x_{i}}\right)=-\operatorname{grad} p+\operatorname{Div} D+F
$$

and transforming the equation we obtain

$$
\frac{\partial v}{\partial t}+\sum_{i=1}^{n} v_{i} \frac{\partial v}{\partial x_{i}}-\mu_{0} \Delta v-C \int_{0}^{t} e^{-\frac{t-s}{\lambda}} \Delta v d s+\operatorname{grad} p=f, \quad \operatorname{div} v=0,
$$

where the vector-function $v$ is connected with the tensor $\left(\varepsilon_{i j}\right)$ as follows:

$$
\varepsilon_{i j}=\frac{1}{2}\left(\frac{\partial v_{i}}{\partial x_{j}}+\frac{\partial v_{j}}{\partial x_{i}}\right), \quad i, j=\overline{1, n} .
$$

It is possible to obtain a model of nonlinear-viscous fluid choosing the nonlinear relation between $D$ and $\mathcal{E}$ in the form

$$
\lambda_{1} \frac{d D}{d t}+D=\varphi_{1}\left(I_{2}\right) \mathcal{E}+\chi_{1} \frac{d}{d t}\left[\psi_{2}\left(I_{2}\right) \mathcal{E}\right] .
$$


Expressing $D$ from this relation

$$
D=\chi_{1} \psi_{2}\left(I_{2}\right) \mathcal{E}+\int_{0}^{t} e^{-\frac{t-s}{\lambda_{1}}}\left[\varphi_{1}\left(I_{2}\right)-\frac{\chi_{1}}{\lambda_{1}} \psi_{2}\left(I_{2}\right)\right] \mathcal{E} d s
$$

and substituting it into the motion equation we obtain

$$
\begin{aligned}
& \frac{\partial v}{\partial t}+\sum_{i=1}^{n} v_{i} \frac{\partial v}{\partial x_{i}}-\mu_{0} \Delta v-\operatorname{Div}\left[2 \mu_{1}\left(I_{2}\right) \mathcal{E}\right]-\int_{0}^{t} e^{-\frac{t-s}{\lambda_{1}}} \operatorname{Div}\left[2 \mu_{2}\left(I_{2}\right) \mathcal{E}\right] d s \\
& \quad+\operatorname{grad} p=f, \quad \operatorname{div} v=0, \quad(x, t) \in Q_{T} .
\end{aligned}
$$

The existence results for strong solutions in the cases $n=2,3$ can be found in [1].

The phenomenological theory of linear visco-elastic fluids with a finite number of discretely distributed times of relaxation and times of retardation uses the relations

$$
\left(1+\sum_{l=1}^{L} \lambda_{l} \frac{d^{l}}{d t^{l}}\right) D=2 \nu\left(1+\sum_{m=1}^{M} \mathfrak{x}_{m} \nu^{-1} \frac{d^{m}}{d t^{m}}\right) \mathcal{E}, \quad \nu, \lambda_{l}, æ_{m}>0 .
$$

For $L=M$ and under additional conditions for coefficients $\left\{\lambda_{l}\right\}, \nu$ and $\left\{æ_{m}\right\}$ (see [8]) the equation of the fluid motion has the following form:

$$
\begin{gathered}
\frac{\partial v}{\partial t}+\sum_{i=1}^{n} v_{i} \frac{\partial v}{\partial x_{i}}-\mu \Delta v-\sum_{l=1}^{L} \beta_{l}^{(0)} \int_{0}^{t} e^{\alpha_{l}(t-s)} \Delta v(s) d s+\operatorname{grad} p=f, \\
(x, t) \in Q_{T}, \quad \operatorname{div} v=0 .
\end{gathered}
$$

In this paper we investigate the above mentioned classes of equations of visco-elastic and nonlinear-viscous fluid motions basing on approximations, using of topological methods for the proof of solvability of approximating problems, and the further limit procedure. It seems that this approach may be useful not only for the solvability but also for the settlement of other questions.

Consider the following initial-boundary value problem for the vectorfunction $v: \bar{Q}_{T} \rightarrow \mathbb{R}^{n}, v=\left(v_{1}, \ldots, v_{n}\right)$, and for the scalar function $p: \bar{Q}_{T} \rightarrow \mathbb{R}:$

$$
\begin{aligned}
\frac{\partial v}{\partial t}- & \mu_{0} \Delta v+\sum_{i=1}^{n} v_{i} \frac{\partial v}{\partial x_{i}}-\operatorname{Div}\left[2 \mu_{1}\left(I_{2}(v)\right) \mathcal{E}(v)\right] \\
& -\int_{0}^{t} \mathcal{L}(t, s) \operatorname{Div}\left[2 \mu_{2}\left(I_{2}(v)\right) \mathcal{E}(v)\right] d s \\
& -\int_{0}^{t} \operatorname{Div} a\left(t, s, x, v(s), D^{1} v(s)\right) d s \\
& +\operatorname{grad} p=f(t, x), \quad(t, x) \in Q_{T} .
\end{aligned}
$$




$$
\begin{gathered}
\operatorname{div} v(t, x)=0, \quad(t, x) \in Q_{T}, \\
v(t, x)=0, \quad x \in \partial \Omega, \quad t \in[0, T], \\
v(0, x)=v^{0}(x), \quad x \in \Omega,
\end{gathered}
$$

where $\mu_{0}>0$ is a constant and $f: Q_{T} \rightarrow \mathbb{R}^{n}, v^{0}: \Omega \rightarrow \mathbb{R}^{n}$ are given functions. Here, and below, $\mathcal{E}(v)$ is a matrix function with components

$$
\varepsilon_{i j}(v)=\frac{1}{2}\left(\frac{\partial v_{i}}{\partial x_{j}}+\frac{\partial v_{j}}{\partial x_{i}}\right) \text { for } i, j=\overline{1, n} \text { and } I_{2}(v)=\left(\sum_{i, j=1}^{n}\left[\varepsilon_{i j}(v)\right]^{2}\right)^{1 / 2} \text {. }
$$

Suppose that the scalar functions $\mu_{i}(s), i=1,2$, are continuously differentiable on $[0,+\infty)$ and satisfy the following conditions:

$\left.\mathbf{M}_{\mathbf{1}}\right) 0 \leq \mu_{i}(s) \leq M$ for all $s \in[0,+\infty)$;

$\left.\mathbf{M}_{\mathbf{2}}\right) s \mu_{i}^{\prime}(s) \leq M$ for all $s \in[0,+\infty)$, and if $\mu_{i}^{\prime}(s)<0$, then $-s \mu_{i}^{\prime}(s) \leq \mu_{i}(s)$.

Note that restrictions for $\mu_{i}, i=1,2$, mentioned above, may be found in $[1],[14]$.

The essentially bounded function $\mathcal{L}(t, s)$ is defined on the set

$$
T d=\{(t, s): t \in[0, T], 0 \leq s \leq t\} .
$$

The matrix function $a(t, s, x, v, w)$ is defined for all $t \in[0, T], 0 \leq s \leq$ $t, x \in \Omega, v \in \mathbb{R}^{n}, w \in \mathbb{R}^{n^{2}}$ and satisfies either the conditions:

$\mathbf{A}_{\mathbf{1}}$ ) the functions $a_{i j}$ (components of $a$ ) are measurable as functions of $t, s, x$ for all $v, w$ and continuous as functions of $v, w$ for almost all $t, s, x$;

A 2$)\left|a_{i j}(t, s, x, v, 0)\right| \leq \mathcal{L}_{1}(t, s, x)+\mathcal{L}_{2}(t, s, x)|v|, i, j=\overline{1, n}$, where $\mathcal{L}_{2}$ is an essentially bounded function and $\mathcal{L}_{1} \in L^{2}(\overline{Q d})$ for $Q d=T d \times \Omega$;

A $\mathbf{A}_{3}\left|a_{i j}(t, s, x, v, w)-a_{i j}(t, s, x, v, \bar{w})\right| \leq \mathcal{L}_{2}(t, s, x)|w-\bar{w}|$ for all possible $t, s, x, v$ and $w, \bar{w} \in \mathbb{R}^{n^{2}}$

or the conditions $\left.\mathbf{A}_{\mathbf{1}}\right)$ and

$\left.\mathbf{A}_{\mathbf{2}}^{\prime}\right)\left|a_{i j}(t, s, x, v, w)-a_{i j}(t, s, x, \bar{v}, \bar{w})\right| \leq \quad \leq \quad \mathcal{L}_{2}(t, s, x)(|v-\bar{v}|+$ $+|w-\bar{w}|)$ for all $t, s, x \in Q d, v, \bar{v} \in \mathbb{R}^{n}, w, \bar{w} \in R^{n^{2}}, i, j=\overline{1, n}$, where $\mathcal{L}_{2}(t, s, x)$ is an essentially-boundary function.

We shall suppose that $n \leq 4$ and $v^{0} \in H, f \in L^{2}((0, T), H)$.

Definition 1.1. A function $v \in L^{2}((0, T), V)$ with $v^{\prime} \in L^{1}\left((0, T), V^{*}\right)$ is said to be a weak solution of the problem (1.1)-(1.4) if for all $h \in V$

$$
\begin{gathered}
\frac{d}{d t} \int_{\Omega} v(t, x) h(x) d x+\mu_{0} \sum_{i=1}^{n} \int_{\Omega} \frac{\partial v}{\partial x_{i}} \cdot \frac{\partial h}{\partial x_{i}} d x-\sum_{i, j=1}^{n} \int_{\Omega} v_{i} v_{j} \frac{\partial h_{j}}{\partial x_{i}} d x \\
+\int_{\Omega} 2 \mu_{1}\left(I_{2}(v)\right) \mathcal{E}(v): \mathcal{E}(h) d x+\int_{0}^{t} \mathcal{L}(t, s) \int_{\Omega} 2 \mu_{2}\left(I_{2}(v)\right) \mathcal{E}(v): \mathcal{E}(h) d x d s
\end{gathered}
$$




$$
+\int_{0}^{t} \int_{\Omega} a\left(t, s, x, v, D^{1} v\right): D^{1} h d x d s=\int_{\Omega} f(t, x) h(x) d x
$$

and

$$
v(0)=v^{0},
$$

where $a: D^{1} h=\sum_{i, j=1}^{n} a_{i j} \cdot \frac{\partial h_{i}}{\partial x_{j}}$ and $\mathcal{E}(v): \mathcal{E}(h)=\sum_{i, j=1}^{n} \varepsilon_{i j}(v) \cdot \varepsilon_{i j}(h)$.

Let us point out that the integral equality (1.5) is obtained from (1.1) by scalar multiplication in $L^{2}(\Omega)$ of each term of (1.1) with $h$ and some simple transformations.

1.3. Statements of main results. Now we formulate the main results for the existence and uniqueness of weak solutions of problem (1.1)-(1.4). Proofs of these results can be found in the fourth section.

Theorem 4.3. Let $n=2$ and the conditions $\left.\left.\left.\left.M_{1}\right)-M_{2}\right), A_{1}\right)-A_{3}\right)$ hold. Then for all $f \in L^{2}((0, T), H)$ and $v^{0} \in H$ there exists at least one weak solution $v \in W$ of problem (1.1)-(1.4) satisfying the following inequalities

$$
\begin{gathered}
\max _{t \in[0, T]}\|v(t)\|_{H}+\sum_{i=1}^{n}\left\|\frac{\partial v}{\partial x_{i}}\right\|_{L^{2}\left(Q_{T}\right)} \leq C\left(1+\|f\|_{L^{2}((0, T), H)}+\left\|v^{0}\right\|_{H}\right), \\
\left\|v^{\prime}\right\|_{L^{2}\left((0, T), V^{*}\right)} \leq C\left(1+\|f\|_{L^{2}((0, T), H)}+\left\|v^{0}\right\|_{H}\right)^{2}
\end{gathered}
$$

with $C$ independent of $v, f, v^{0}$.

Theorem 4.4. Let $n=2$ and the conditions $\left.\left.\left.M_{1}\right)-M_{2}\right), A_{1}\right)-A_{2}^{\prime}$ ) hold. Then for all $f \in L^{2}((0, T), H), v^{0} \in H$ the weak solution $v \in W$ of problem (1.1)-(1.4) is unique.

In the case $2 \leq n \leq 4$ we establish existence of a weak solution for equations of the form:

$$
\begin{aligned}
& \frac{\partial v}{\partial t}-\mu_{0} \Delta v+\sum_{i=1}^{n} v_{i} \frac{\partial v}{\partial x_{i}}-\int_{0}^{t} \operatorname{Div}\left(a\left(t, s, x, v(s, x), D^{1} v(s, x)\right) d s\right. \\
& \quad+\operatorname{grad} p=f, \quad(t, x) \in Q_{T}
\end{aligned}
$$

where the elements of the matrix-function $a$ are defined by

$$
\begin{gathered}
a_{i j}\left(t, s, x, v(s, x), D^{1} v(s, x)\right) \\
=b(i, j ; t, s, x): D^{1} v(s, x)+c(i, j ; t, s, x) \cdot v(s, x) .
\end{gathered}
$$

Theorem 4.5. Let $2 \leq n \leq 4$ and assume that the matrix functions $b(i, j, \cdot)$ and the vector functions $c(i, j, \cdot)$ are essentially bounded for $i, j=\overline{1, n}$. Then for all $f \in L^{2}((0, T), H)$ and $v^{0} \in H$ there exists at least one weak solution

$$
v \in L^{2}((0, T), V) \text { with } v^{\prime} \in L^{1}\left((0, T), V^{*}\right)
$$


of problem (1.7), (1.2)-(1.4), which satisfies the following inequalities:

$$
\begin{gathered}
\max _{t \in[0, T]}\|v(t)\|_{H}+\sum_{i=1}^{n}\left\|\frac{\partial v}{\partial x_{i}}\right\|_{L^{2}\left(Q_{T}\right)} \leq C\left(1+\|f\|_{L^{2}((0, T), H)}+\left\|v^{0}\right\|_{H}\right), \\
\left\|v^{\prime}\right\|_{L^{1}\left((0, T), V^{*}\right)} \leq C\left(1+\|f\|_{L^{2}((0, T), H)}+\left\|v^{0}\right\|_{H}\right)^{2}
\end{gathered}
$$

with $C$ independent of $v, f$ and $v^{0}$.

\section{Operator AND APPROXIMATING EQUATIONS}

In this section we introduce operator equations which are equivalent to the problem of weak solutions of (1.5)-(1.6), and then we construct a family of approximating equations and investigate properties of the operators involved.

\subsection{The operator equation which is equivalent to the weak solu-} tions problem. Let $v$ be a weak solution of the problem (1.1)-(1.4). Then the function $v$ satisfies (1.5) for all $h \in V$. Taking into account identifications

$$
V \subseteq H \equiv H^{*} \subset V^{*},
$$

consider each term of (1.5) as the action of some functional on the function $h$. Thus

$$
\int_{\Omega} f \cdot h d x=(f, h)=\langle f, h\rangle \text { for } h \in V,
$$

where $f$ is considered as an element of the space $L^{2}\left((0, T), V^{*}\right)$. Suppose for all $t \in[0, T]$

$$
\begin{aligned}
& \sum_{i=1}^{n} \int_{\Omega} \frac{\partial v}{\partial x_{i}} \cdot \frac{\partial h}{\partial x_{i}} d x=\langle A v, h\rangle, \\
& \int_{\Omega} 2 \mu_{i}\left(I_{2}(v)\right) \mathcal{E}(v): \mathcal{E}(h) d x=\left\langle B_{i}(v), h\right\rangle, \quad i=1,2, \\
& \int_{\Omega} a\left(t, s, x, v, D^{1} v\right): D^{1} h d x=-\langle G(t, s, v), h\rangle, \\
& \sum_{i, j=1}^{n} \int_{\Omega} v_{i} v_{j} \frac{\partial h_{j}}{\partial x_{i}} d x=\langle K(v), h\rangle, \\
& \frac{d}{d t} \int_{\Omega} v \cdot h d x=\frac{d}{d t}\langle v, h\rangle=\left\langle v^{\prime}, h\right\rangle .
\end{aligned}
$$

The last equality follows from [15, Lemma 1.1.]. Taking into account the above notations we can rewrite identity (1.5) in the form:

$$
\begin{aligned}
\left\langle v^{\prime}, h\right\rangle & +\mu_{0}\langle A v, h\rangle-\langle K(v), h\rangle+\left\langle B_{1}(v), h\right\rangle \\
& +\int_{0}^{t} \mathcal{L}(t, s)\left\langle B_{2}(v(s)), h\right\rangle d s-\int_{0}^{t}\langle G(t, s, v(s)), h\rangle d s=\langle f, h\rangle
\end{aligned}
$$


for $\forall h \in V$ and for almost all $t \in[0, T]$.

Lemma 2.1. Let $n \leq 4$ and the conditions $\left.\left.\left.M_{1}\right)-M_{2}\right), A_{1}\right)-A_{3}$ ) hold. Then

1) for every function $v \in L^{2}((0, T), V)$ functions $A v, B_{i}(v), \quad i=1,2$, $C(v)=\int_{0}^{t} \mathcal{L}(t, s) B_{2}(v(s)) d s$ and $Q(v)=\int_{0}^{t} G(t, s, v(s)) d s$ belong to the space $L^{2}\left((0, T), V^{*}\right) ; G(t, s, v(s))$ belongs to the space $L^{2}\left(T d, V^{*}\right) ; K(v)$ belongs to the space $L^{1}\left((0, T), V^{*}\right)$;

2) operators $A, B_{1}, B_{2}, C, Q: X \rightarrow X^{*}$ and $K: X \rightarrow L^{1}\left((0, T), V^{*}\right)$ are continuous;

3) the following estimates are valid:

$$
\begin{gathered}
\|A v\|_{X^{*}} \leq C\left(1+\|v\|_{X}\right), \\
\left\|B_{i}(v)\right\|_{X^{*}} \leq C\left(1+\|v\|_{X}\right), \quad i=1,2, \\
\|C(v)\|_{X^{*}} \leq C\left(1+\|v\|_{X}\right), \\
\|Q(v)\|_{X^{*}} \leq C\left(1+\|v\|_{X}\right), \\
\|K(v)\|_{L^{1}\left((0, T), V^{*}\right)} \leq C\|v\|_{X}^{2},
\end{gathered}
$$

for all $v \in X$, and $C$ is a constant depending only on characteristic constants and functions included in conditions $\left.\left.\left.A_{1}\right)-A_{3}\right), M_{1}\right)-M_{2}$ ).

Proof. 1) Consider the function $G(t, s, v(s))$. By definition

$$
\langle G(t, s, v(s)), h\rangle=-\int_{\Omega} a\left(t, s, x, v(s, x), D^{1} v(s, x)\right): D^{1} h(x) d x
$$

for every $h \in V$. Therefore

$$
\begin{gathered}
\|G(t, s, v(s))\|_{V^{*}} \leq\left\|a\left(t, s, x, v(s, x), D^{1} v(s, x)\right)\right\|_{H} \\
\leq\left\|\mathcal{L}_{1}(t, s, x)\right\|_{H}+\left\|\mathcal{L}_{2}\right\|_{L^{\infty}(Q d)}\|v\|_{H}+\left\|\mathcal{L}_{2}\right\|_{L^{\infty}(Q d)}\left\|D^{1} v\right\|_{H}
\end{gathered}
$$

by conditions $\left.\left.A_{2}\right)-A_{3}\right)$. We rewrite the inequality in the form

$$
\|G(t, s, v(s))\|_{V^{*}} \leq\left\|\mathcal{L}_{1}(t, s, \cdot)\right\|_{H}+\left\|\mathcal{L}_{2}(t, s, \cdot)\right\|_{L^{\infty}(\Omega)} \cdot\|v(s)\|_{V}
$$

with some constant $C$. Note that functions $\left\|\mathcal{L}_{1}\right\|_{H}$ and $\|v\|_{V}$ are square integrable on $T d$ and, hence, the function $G(t, s, v(s))$ belongs to $L^{2}\left(T d, V^{*}\right)$. Then

$$
\begin{gathered}
\left\|\int_{0}^{t} G(t, s, v(s)) d s\right\|_{V^{*}} \leq \int_{0}^{t}\|G(t, s, v(s))\|_{V^{*}} d s \\
\leq \int_{0}^{t}\left(\left\|\mathcal{L}_{1}(t, s, x)\right\|_{H}+C\|v(s)\|_{V}\right) d s \leq \int_{0}^{t}\left\|\mathcal{L}_{1}(t, s, x)\right\|_{H} d s+C\|v\|_{X} .
\end{gathered}
$$


By assumption $A_{2}$ ), the right-hand side of the inequality is square integrable in the variable $t$. Hence, the function $Q(v)=\int_{0}^{t} G(t, s, v(s)) d s$ belongs to the space $L^{2}\left((0, T), V^{*}\right)$ and

$$
\|Q(v)\|_{X^{*}} \leq\left(\int_{0}^{T}\left\|\int_{0}^{t} G(t, s, v(s)) d s\right\|_{V^{*}}^{2} d t\right)^{1 / 2} \leq C\left(1+\|v\|_{X}\right),
$$

where $C$ depends only on $\left\|\mathcal{L}_{1}\right\|_{L^{2}(Q d)}$ and $\left\|\mathcal{L}_{2}\right\|_{L^{\infty}(Q d)}$.

2) To prove the continuity of the map

$$
G: X \rightarrow L^{2}\left(T d, V^{*}\right), \quad v \mapsto G(t, s, v(s)),
$$

it is sufficient to show the continuity of the map

$$
a: X \rightarrow L^{2}(Q d), \quad v \mapsto a\left(t, s, x, v(s, x), D^{1} v(s, x)\right) .
$$

It is known [9] that under assumptions $\left.A_{1}\right)-A_{3}$ ) the Nemytskii operator $a$ is continuous. Hence, the map $G$ is continuous too. Thus, $Q$ is continuous as a composition of two continuous maps, namely, $G$ and the integral operator.

By similar arguments one can check that the definition is well-defined, prove that the maps $A, B_{1}, B_{2}, C$ are continuous and obtain the estimates for them.

3) Consider the function $K(v)$. By definition,

$$
\langle K(v), h\rangle=\sum_{i, j=1}^{n} \int_{\Omega} v_{i} v_{j} \frac{\partial h_{j}}{\partial x_{i}} d x .
$$

Therefore $\|K(v)\|_{V^{*}} \leq C \max _{i j}\left\|v_{i} v_{j}\right\|_{H} \leq C\|v\|_{L^{4}(\Omega)}^{2}$. By Sobolev's embedding theorem [6], we have the continuous embedding $V \subset L^{4}(\Omega)$ when $n \leq 4$ and, hence,

$$
\|v\|_{L^{4}(\Omega)} \leq C\|v\|_{V} \text { and }\|K(v)\|_{V^{*}} \leq C\|v\|_{V}^{2} .
$$

Thus, $\|K(v)\|_{L^{1}\left((0, T), V^{*}\right)} \leq C\|v\|_{X}^{2}$. The continuity of $K$ follows from the continuity of the embedding $X \subset L^{2}\left((0, T), L^{4}(\Omega)\right)$ and the continuity of the Nemytskii operators

$$
k_{i j}: L^{2}\left((0, T), L^{4}(\Omega)\right) \rightarrow L^{1}\left((0, T), L^{2}(\Omega)\right), \quad k_{i j}(v)=v_{i} v_{j} .
$$

By $[6$, Theorem 8],

$$
\int_{0}^{t} \mathcal{L}(t, s)\left\langle B_{2}(v(s)), h\right\rangle d s=\left\langle\int_{0}^{t} \mathcal{L}(t, s) B_{2}(v(s)) d s, h\right\rangle
$$

and

$$
\int_{0}^{t}\langle G(t, s, v(s)), h\rangle d s=\left\langle\int_{0}^{t} G(t, s, v(s)) d s, h\right\rangle
$$


Hence, applying lemma 2.1, we rewrite the equality (2.1) in the form:

$$
\begin{aligned}
v^{\prime}+\mu_{0} A v & -K(v)+B_{1}(v)+\int_{0}^{t} \mathcal{L}(t, s) B_{2}(v(s)) d s \\
& -\int_{0}^{t} G(t, s, v(s)) d s=f .
\end{aligned}
$$

It follows that every weak solution of problem (1.1)-(1.4) is a solution of the operator equation (2.3) with

$$
v(0)=v^{0}
$$

Repeating arguments ([15], p. 226), it is easy to show that the equality (2.4) makes sense and every solution of problem (2.3)-(2.4) is a weak solution of problem (1.1)-(1.4).

2.2. Approximating equations. To investigate the solvability of the operator equation (2.3) we introduce (following [2], [14]) nonlinear approximating equations.

We replace the nonlinear term

$$
\sum_{i=1}^{n} v_{i} \frac{\partial v}{\partial x_{i}}
$$

in (1.1) by the term

$$
\sum_{i=1}^{n} \frac{\partial}{\partial x_{i}}\left(\frac{v_{i} v}{1+\varepsilon|v|^{2}}\right)
$$

with $\varepsilon>0$, and obtain the equation

$$
\begin{aligned}
\frac{\partial v}{\partial t}- & \mu_{0} \Delta v+\sum_{i=1}^{n} \frac{\partial}{\partial x_{i}}\left(\frac{v_{i} v}{1+\varepsilon|v|^{2}}\right)-\operatorname{Div}\left[2 \mu_{1}\left(I_{2}(v)\right) \mathcal{E}(v)\right] \\
& -\int_{0}^{t} \mathcal{L}(t, s) \operatorname{Div}\left[2 \mu_{2}\left(I_{2}(v)\right) \mathcal{E}(v)\right] d s \\
& -\int_{0}^{t} \operatorname{Diva}\left(t, s, x, v(s, x), D^{1} v(s, x)\right) d s \\
& +\operatorname{grad} p=f(t, x), \quad(x, t) \in Q_{T} .
\end{aligned}
$$


Repeating above arguments for equation $\left(1.1_{\varepsilon}\right)$ instead of (1.1), we obtain that the weak solutions of problem $\left(1.1_{\varepsilon}\right)-(1.4)$ are solutions of the approximating operator equation

$$
\begin{aligned}
& v^{\prime}+\mu_{0} A v-D_{\varepsilon}(v)+B_{1}(v)+\int_{0}^{t} \mathcal{L}(t, s) B_{2}(v(s)) d s \\
& -\int_{0}^{t} G(t, s, v(s)) d s=f, \quad \varepsilon>0,
\end{aligned}
$$

with $v(0)=v^{0}$. And vice versa, any solution of problem $\left(2.3_{\varepsilon}\right),(2.4)$ is a weak solution of problem $\left(1.1_{\varepsilon}\right)-(1.4)$.

The functional $D_{\varepsilon}(v)$ used in the equality $\left(2.3_{\varepsilon}\right)$ is defined by

$$
\sum_{i, j=1}^{n} \int_{\Omega} \frac{v_{i} v_{j}}{1+\varepsilon|v|^{2}} \cdot \frac{\partial h_{j}}{\partial x_{i}} d x=\left\langle D_{\varepsilon}(v), h\right\rangle, \quad h \in V .
$$

Since

and

$$
\left|\frac{v_{i} v_{j}}{1+\varepsilon|v|^{2}}\right| \leq \frac{1}{\varepsilon}
$$

we get

Hence, $D_{\varepsilon}(v) \in L^{\infty}\left((0, T), V^{*}\right)$ and

$$
\left\|D_{\varepsilon}(v)\right\|_{V^{*}} \leq C \max _{i, j}\left\|\frac{v_{i} v_{j}}{1+\varepsilon|v|^{2}}\right\|_{H}
$$

$$
\left\|D_{\varepsilon}(v)\right\|_{X^{*}} \leq \frac{C}{\varepsilon}
$$

Moreover, the map $D_{\varepsilon}: X \rightarrow X^{*}$ is continuous since it is a Nemytskii operator.

Note that, for $v \in X$, all the terms in $\left(2.3_{\varepsilon}\right)$ (but the first one) belong to the space $X^{*}$. Therefore, for a solution $v$ of $\left(2.3_{\varepsilon}\right)$ we get $v^{\prime} \in X^{*}$. Hence, any solution belongs to the space $W=\left\{v: v \in X, v^{\prime} \in X^{*}\right\}$. It is known [6, Theorem 1.16] that the space $W$ is Banach and the embedding $W \subset$ $C([0, T], H)$ is continuous [6, Theorem 1.17]. Thus, the operator $\left.v \mapsto v\right|_{t=0}$ is well defined on $W$, takes values in $H$ and is continuous.

Let us introduce the following notations.

$$
\begin{aligned}
& \mathcal{A}: W \rightarrow X^{*} \times H, \quad \mathcal{A}(v)=\left(v^{\prime}+\mu_{0} A v+B_{1}(v)+C(v),\left.v\right|_{t=0}\right) ; \\
& g: W \subseteq X \rightarrow X^{*} \times H, \quad g(v)=(Q(v), 0), \\
& K_{\varepsilon}: W \subset X \rightarrow X^{*} \times H, \quad K_{\varepsilon}(v)=\left(D_{\varepsilon}(v), 0\right) .
\end{aligned}
$$

It is easy to see that problem $\left(2.3_{\varepsilon}\right),(2.4)$ is equivalent to the operator equation

$$
\mathcal{A}(v)-K_{\varepsilon}(v)-g(v)=\left(f, v^{0}\right) .
$$

It follows that the problem of weak solutions of $\left(1.1_{\varepsilon}\right)-(1.4)$ is equivalent to the problem of the solvability of the operator equation $\left(2.6_{\varepsilon}\right)$. 
We shall now investigate the properties of the operators $\mathcal{A}, K_{\varepsilon}$ and $g$ appearing in $\left(2.6_{\varepsilon}\right)$.

2.3. Properties of the operator $\mathcal{A}$. W first study the properties of the map $\mathcal{A}$. Then we show that $\mathcal{A}$ is an invertible map and its inverse $\mathcal{A}^{-1}$ is a contraction.

Lemma 2.2. If the functions $\mu_{i}(s)$ satisfy the assumptions $\left.M_{1}\right)-M_{2}$ ), then, for all $u, v \in V$,

$$
\begin{gathered}
\left\langle B_{i}(u)-B_{i}(v), u-v\right\rangle \geq 0, \\
\left\langle B_{i}(u)-B_{i}(v), u-v\right\rangle \leq C(M)\|u-v\|_{V}^{2}, i=1,2,
\end{gathered}
$$

where $C(M)$ is a constant depending on $M$ from conditions $\left.M_{1}\right)-M_{2}$ ).

This statement is well known. For example, it was used in [1]. We give its proof for completeness.

Proof. Let $u, v \in V$. By the definition of $B_{i}$,

$$
\begin{gathered}
\left\langle B_{i}(u)-B_{i}(v), u-v\right\rangle \\
=\int_{\Omega}\left(2 \mu_{i}\left(I_{2}(u)\right) \cdot \mathcal{E}(u)-2 \mu_{i}\left(I_{2}(v)\right) \cdot \mathcal{E}(v)\right):(\mathcal{E}(u)-\mathcal{E}(v)) d x .
\end{gathered}
$$

Using the mean value theorem for integrals we write this expression as follows:

$$
\begin{aligned}
& 2 \int_{\Omega} \int_{0}^{1} \frac{d}{d s}\left(\mu_{i}\left(I_{2}(v+s(u-v))\right) \mathcal{E}(v+s(u-v))\right) d s: \mathcal{E}(u-v) d x \\
& =2 \int_{\Omega} \int_{0}^{1}\left(\mu_{i}\left(I_{2}(v+s(u-v))\right) \mathcal{E}(u-v)\right. \\
& \left.\quad+\frac{d \mu_{i}\left(I_{2}(v+s(u-v))\right)}{d s} \cdot \mathcal{E}(v+s(u-v))\right) d s: \mathcal{E}(u-v) d x \\
& =2 \int_{\Omega}\left(\mu_{i}\left(I_{2}\left(v+s_{0}(u-v)\right)\right) \mathcal{E}(u-v): \mathcal{E}(u-v)\right. \\
& \quad+\mu_{i}^{\prime}\left(I_{2}\left(v+s_{0}(u-v)\right)\right) \frac{\mathcal{E}\left(v+s_{0}(u-v)\right): \mathcal{E}(u-v)}{I_{2}\left(v+s_{0}(u-v)\right)} \\
& \left.\quad \cdot \mathcal{E}\left(v+s_{0}(u-v)\right): \mathcal{E}(u-v)\right) d x \\
& =2 \int_{\Omega}\left(\mu_{i}\left(I_{2}\left(v+s_{0}(u-v)\right)\right) \mathcal{E}(u-v): \mathcal{E}(u-v)\right. \\
& \left.\quad+\frac{\mu_{i}^{\prime}\left(I_{2}\left(v+s_{0}(u-v)\right)\right)}{I_{2}\left(v+s_{0}(u-v)\right)} \cdot\left(\mathcal{E}\left(v+s_{0}(u-v)\right): \mathcal{E}(u-v)\right)^{2}\right) d x .
\end{aligned}
$$

Observe that if $\mu_{i}^{\prime}\left(I_{2}\left(v+s_{0}(u-v)\right)\right) \geq 0$, then the second term is nonnegative. Since $\mu_{i}(s) \geq 0$, the first term is also nonnegative. Thus, the integrand is nonnegative. 
In the case $\mu_{i}^{\prime}\left(I_{2}\left(v+s_{0}(u-v)\right)\right)<0$ we use the inequality

$$
\begin{gathered}
\left(\mathcal{E}\left(v+s_{0}(u-v)\right): \mathcal{E}(u-v)\right)^{2} \\
\leq\left(\mathcal{E}\left(v+s_{0}(u-v)\right): \mathcal{E}\left(v+s_{0}(u-v)\right)\right) \cdot(\mathcal{E}(u-v): \mathcal{E}(u-v))
\end{gathered}
$$

and the relation

$$
\mathcal{E}\left(v+s_{0}(u-v)\right): \mathcal{E}\left(v+s_{0}(u-v)\right)=\left(I_{2}\left(v+s_{0}(u-v)\right)\right)^{2} .
$$

Then

$$
\begin{aligned}
& \mu_{i}\left(I_{2}\left(v+s_{0}(u-v)\right)\right) \mathcal{E}(u-v): \mathcal{E}(u-v) \\
& \left.\quad+\frac{\mu_{i}^{\prime}\left(I_{2}\left(v+s_{0}(u-v)\right)\right)}{\left.I_{2}\left(v+s_{0}(u-v)\right)\right)} \cdot\left(\mathcal{E}\left(v+s_{0}(u-v)\right)\right): \mathcal{E}(u-v)\right)^{2} \\
& \geq\left(\mu_{i}\left(I_{2}\left(v+s_{0}(u-v)\right)\right)+I_{2}\left(v+s_{0}(u-v)\right)\right) \\
& \left.\quad \cdot \mu_{i}^{\prime}\left(I_{2}\left(v+s_{0}(u-v)\right)\right)\right) \mathcal{E}(u-v): \mathcal{E}(u-v) .
\end{aligned}
$$

This expression is nonnegative since $\mu_{i}(s)+s \mu_{i}^{\prime}(s) \geq 0$ for $\mu^{\prime}(s)<0$.

We have actually proved that the integrand is nonnegative. Hence,

$$
\left\langle B_{i}(u)-B_{i}(v), u-v\right\rangle \geq 0 .
$$

Using the above relations and inequalities we can similarly get the estimate

$$
\begin{aligned}
& \left\langle B_{i}(u)-B_{i}(v), u-v\right\rangle \\
& \leq 2 \int_{\Omega}\left(\left|\mu_{i}\left(I_{2}\left(v+s_{0}(u-v)\right)\right)\right| \cdot \varepsilon(u-v): \varepsilon(u-v)\right. \\
& \left.\left.\quad+\frac{\left|\mu_{i}^{\prime}\left(I_{2}\left(v+s_{0}(u-v)\right)\right)\right|}{I_{2}\left(v+s_{0}(u-v)\right)} \cdot\left(\mathcal{E}\left(v+s_{0}(u-v)\right)\right): \mathcal{E}(u-v)\right)^{2}\right) d x \\
& \leq 2 \int_{\Omega}\left(\left|\mu_{i}\left(I_{2}\left(v+s_{0}(u-v)\right)\right)\right|+I_{2}\left(v+s_{0}(u-v)\right)\right. \\
& \left.\quad \cdot\left|\mu^{\prime}\left(I_{2}\left(v+s_{0}(u-v)\right)\right)\right|\right) \cdot \mathcal{E}(u-v): \mathcal{E}(u-v) d x \\
& \leq 4 M \int_{\Omega} \sum_{i, j=1}^{n}\left(\varepsilon_{i j}(u-v)\right)^{2} d x \leq C(M)\|u-v\|_{V}^{2} \cdot \cdot
\end{aligned}
$$

As we mentioned above, $W \subset C([0, T], H)$. hence, $W \subset X \cap C([0, T], H)$. For functions $v \in X \cap C([0, T], H)$, we consider the norm

$$
\|v\|_{X C}=\max _{0 \leq t \leq T}\|v(t)\|_{H}+\sum_{i=1}^{n}\left\|\frac{\partial v}{\partial x_{i}}\right\|_{L^{2}((0, T), H)}
$$

and the equivalent norms

$$
\|v\|_{k, X C}=\left\|e^{-k t} v(t)\right\|_{X C} \text { for } k>0 .
$$

Similarly, we define equivalent norms $\|\cdot\|_{k, X},\|\cdot\|_{k, X^{*} \times H},\|\cdot\|_{k, L^{2}((0, T), H)}$ for the spaces $X, X^{*} \times H$ and $L^{2}((0, T), H)=L^{2}\left(Q_{T}\right)$, respectively. 
Lemma 2.3. If $\mu_{2}(s)$ satisfies the assumptions $\left.\left.M_{1}\right)-M_{2}\right)$, then for all $u, v \in W$ and $k>0$,

$$
\int_{0}^{T} e^{-2 k t}\langle C(v)-C(u), v-u\rangle d t \leq \frac{C}{\sqrt{2 k}}\|v-u\|_{k, X}^{2}
$$

where $C$ is independent of $u, v$ and $k$.

Proof. Let $u, v \in X$. By the definitions of the operators $C$ and $B_{2}$,

$$
\begin{aligned}
& \langle C(v)-C(u), v-u\rangle \\
& =\int_{0}^{t} \mathcal{L}(t, s)\left\langle B_{2}(v(s))-B_{2}(u(s)), v(t)-u(t)\right\rangle d s \\
& =\int_{0}^{t} \mathcal{L}(t, s) \int_{\Omega}\left(2 \mu_{2}\left(I_{2}(v(s))\right) \mathcal{E}(v(s))-2 \mu_{2}\left(I_{2}(u(s))\right) \mathcal{E}(u(s))\right) \\
& \quad:(\mathcal{E}(v(t))-\mathcal{E}(u(t))) d x d s .
\end{aligned}
$$

Using the mean value theorem for integrals we get

$$
\begin{aligned}
2 & \int_{0}^{t} \mathcal{L}(t, s) \int_{\Omega} \int_{0}^{1} \frac{d}{d \tau}\left(\mu_{2}\left(I_{2}(u(s)+\tau(v(s)-u(s)))\right) \mathcal{E}(u(s)\right. \\
& +\tau(v(s)-u(s)))) d \tau: \mathcal{E}(v(t)-u(t)) d x d s \\
= & 2 \int_{0}^{t} \mathcal{L}(t, s) \int_{\Omega} \int_{0}^{1}\left(\mu_{2}\left(I_{2}(u(s)+\tau(v(s)-u(s)))\right) \cdot \mathcal{E}(v(s)-u(s))\right. \\
& +\frac{d \mu_{2}\left(I_{2}(u(s)+\tau(v(s)-u(s)))\right)}{d \tau} \\
& \cdot \mathcal{E}(u(s)+\tau(v(s)-u(s)))) d \tau:(\mathcal{E}(v(t)-u(t))) d x d s \\
= & 2 \int_{0}^{t} \mathcal{L}(t, s) \int_{\Omega}\left(\mu_{2}\left(I_{2}\left(u(s)+\tau_{0}(v(s)-u(s))\right)\right) \mathcal{E}(v(s)\right. \\
& -u(s)): \mathcal{E}(v(t)-u(t)) \\
& +\frac{\mu_{2}^{\prime}\left(I_{2}\left(u(s)+\tau_{0}(v(s)-u(s))\right)\right)}{I_{2}\left(u(s)+\tau_{0}(v(s)-u(s))\right)} \cdot \mathcal{E}\left(u(s)+\tau_{0}(v(s)-u(s))\right) \\
& \left.: \mathcal{E}(v(s)-u(s)) \cdot \mathcal{E}\left(u(s)+\tau_{0}(v(s)-u(s))\right): \mathcal{E}(v(t)-u(t))\right) d x d s .
\end{aligned}
$$


By the Cauchy inequality,

$$
\begin{aligned}
& |\mathcal{E}(v(s)-u(s)): \mathcal{E}(v(t)-u(t))| \\
& \leq I_{2}(v(s)-u(s)) \cdot I_{2}(v(t)-u(t)), \\
& \left|\mathcal{E}\left(u(s)+\tau_{0}(v(s)-u(s))\right): \mathcal{E}(v(s)-u(s))\right| \\
& \leq I_{2}\left(u(s)+\tau_{0}(v(s)-u(s))\right) \cdot I_{2}(v(s)-u(s)), \\
& \left|\mathcal{E}\left(u(s)+\tau_{0}(v(s)-u(s))\right): \mathcal{E}(v(t)-u(t))\right| \\
& \leq I_{2}\left(u(s)+\tau_{0}(v(s)-u(s))\right) \cdot I_{2}(v(t)-u(t)) .
\end{aligned}
$$

Hence,

$$
\begin{aligned}
& \langle C(v)-C(u), v-u\rangle \leq 2 \int_{0}^{t} \mathcal{L}(t, s) \int_{\Omega}\left(\mid \mu_{2}\left(I_{2}(u(s)\right.\right. \\
& \left.\left.\quad+\tau_{0}(v(s)-u(s))\right)\right) \mid \cdot I_{2}(v(s)-u(s)) \cdot I_{2}(v(t)-u(t)) \\
& \quad+\mid \mu_{2}^{\prime}\left(I_{2}\left(u(s)+\tau_{0}(v(s)-u(s))\right)\right) \cdot I_{2}(u(s) \\
& \left.\quad+\tau_{0}(v(s)-u(s))\right) \cdot I_{2}(v(s)-u(s)) \cdot I_{2}(v(t)-u(t)) d x d s \\
& \leq 4 M \int_{0}^{t} \mathcal{L}(t, s) \int_{\Omega} I_{2}(v(s)-u(s)) \cdot I_{2}(v(t)-u(t)) d x d s .
\end{aligned}
$$

Let us consider the functions $\bar{u}(t)=e^{-k t} u(t)$ and $\bar{v}(t)=e^{-k t} v(t)$. It is obvious that $\|u\|_{k, X}=\|\bar{u}\|_{X}$ and $\|v\|_{k, X}=\|\bar{v}\|_{X}$. By the Hölder inequality we obtain

$$
\begin{aligned}
& \int_{0}^{T} e^{-2 k t}\langle C(v(t))-C(u(t)), v(t)-u(t)\rangle d t \\
& \leq 4 M\|\mathcal{L}\|_{L^{\infty}(T d)} \int_{0}^{T} e^{-2 k t} \int_{0}^{t}\|\mathcal{E}(v(s)-u(s))\|_{L^{2}(\Omega)} \\
& \cdot\|\mathcal{E}(v(t)-u(t))\|_{L^{2}(\Omega)} d s d t=4 M\|\mathcal{L}\|_{L^{\infty}(T d)} \int_{0}^{T}\|\mathcal{E}(\bar{v}(t)-\bar{u}(t))\|_{L^{2}(\Omega)} \\
& \quad \int_{0}^{t} e^{-k(t-s)}\|\mathcal{E}(\bar{v}(s)-\bar{u}(s))\|_{L^{2}(\Omega)} d s d t \\
& \leq 4 M\|\mathcal{L}\|_{L^{\infty}(T d)} \cdot \int_{0}^{T}\|\mathcal{E}(\bar{v}(t)-\bar{u}(t))\|_{L^{2}(\Omega)}
\end{aligned}
$$




$$
\begin{gathered}
\cdot\left(\int_{0}^{t}\|\mathcal{E}(\bar{v}(s)-\bar{u}(s))\|_{L^{2}(\Omega)}^{2} d s\right)^{1 / 2} \cdot\left(\int_{0}^{t} e^{-2 k(t-s)} d s\right)^{1 / 2} d t \\
\leq 4 M\|\mathcal{L}\|_{L^{\infty}(T d)}\|\mathcal{E}(\bar{v}-\bar{u})\|_{L^{2}\left(Q_{T}\right)}^{2} \cdot\left(\int_{0}^{T} \int_{0}^{t} e^{-2 k(t-s)} d s d t\right)^{1 / 2} .
\end{gathered}
$$

As

$$
\begin{aligned}
\int_{0}^{T} \int_{0}^{t} e^{-2 k(t-s)} d s d t & =\frac{1}{2 k} \int_{0}^{T}\left(1-e^{-2 k t}\right) d t \\
& =\frac{1}{2 k}\left(T+\frac{1}{2 k}\left(e^{-2 k T}-1\right)\right) \leq \frac{T}{2 k}
\end{aligned}
$$

we have

$$
\begin{aligned}
& \int_{0}^{T} e^{-2 k t}\langle C(v(t))-C(u(t)), v(t)-u(t)\rangle d t \\
& \leq \frac{4 M\|\mathcal{L}\|_{L^{\infty}(T d)}}{\sqrt{2 k}} \sqrt{T}\|\mathcal{E}(\bar{v}-\bar{u})\|_{L^{2}(\Omega)}^{2} \\
& \leq \frac{C}{\sqrt{2 k}}\|\bar{v}-\bar{u}\|_{X}^{2}=\frac{C}{\sqrt{2 k}}\|v-u\|_{k, X}^{2} \cdot \bullet
\end{aligned}
$$

Consider the auxiliary problem

$$
\begin{aligned}
& v^{\prime}+\mu_{0} A v+B_{1}(v)+C(v)=\varphi, \quad \varphi \in X^{*}, \\
& \left.v\right|_{t=0}=a .
\end{aligned}
$$

Letting $v(t)=e^{k t} \bar{v}(t), \varphi=e^{k t} \bar{\varphi}(t)$ and multiplying by $e^{-k t}$ we obtain

$$
\begin{aligned}
& \bar{v}^{\prime}+k \bar{v}+\mu_{0} A \bar{v}+e^{-k t} B_{1}\left(e^{k t} \bar{v}(t)\right)+e^{-k t} C\left(e^{k t} \bar{v}\right)=\varphi, \\
& \left.\bar{v}\right|_{t=0}=a .
\end{aligned}
$$

Lemma 2.4. If functions $\mu_{i}(s)$ satisfy the conditions $\left.\left.M_{1}\right)-M_{2}\right)$, then the operator $V_{k}: X \rightarrow X^{*}$, defined by the equality

$$
V_{k}(\bar{v})=k \bar{v}+\mu_{0} A \bar{v}+e^{-k t} B_{1}\left(e^{k t} \bar{v}\right)+e^{-k t} C\left(e^{k t} \bar{v}\right)
$$

is continuous, monotone and coercive for $k$ large enough.

Proof. The continuity of the operator follows from the continuity of each term. 
Let us show the monotonicity of the operator $V_{k}$. For arbitrary functions $\bar{u}, \bar{v} \in X$, we have

$$
\begin{aligned}
& \int_{0}^{T}\left\langle V_{k}(\bar{v}(t))-V_{k}(\bar{u}(t)), \bar{v}(t)-\bar{u}(t)\right\rangle d t \\
& =k \int_{0}^{T}\|\bar{v}(t)-\bar{u}(t)\|_{H}^{2} d t+\mu_{0} \int_{0}^{T}((\bar{v}(t)-\bar{u}(t), \bar{v}(t)-\bar{u}(t))) d t \\
& \quad+\int_{0}^{T} e^{-k t}\left\langle B_{1}\left(e^{k t} \bar{v}(t)\right)-B_{1}\left(e^{k t} \bar{u}(t)\right), \bar{v}(t)-\bar{u}(t)\right\rangle d t \\
& \quad+\int_{0}^{T} e^{-k t}\left\langle C\left(e^{k t} \bar{v}(t)\right)-C\left(e^{k t} \bar{u}(t)\right), \bar{v}(t)-\bar{u}(t)\right\rangle d t .
\end{aligned}
$$

We evaluate terms at the right hand side of the equation. For $k>\mu_{0}$,

$$
k \int_{0}^{T}\|\bar{v}(t)-\bar{u}(t)\|_{H}^{2} d t+\mu_{0} \int_{0}^{T}((\bar{v}(t)-\bar{u}(t), \bar{v}(t)-\bar{u}(t))) d t \geq \mu_{0}\|\bar{v}-\bar{u}\|_{X}^{2} .
$$

Applying lemma 2.2 we have

$$
\begin{aligned}
& \int_{0}^{T} e^{-k t}\left\langle B_{1}\left(e^{k t} \bar{v}(t)\right)-B_{1}\left(e^{k t} \bar{u}(t)\right), \bar{v}(t)-\bar{u}(t)\right\rangle d t \\
& =\int_{0}^{T} e^{-2 k t}\left\langle B_{1}(v(t))-B_{1}(u(t)), v(t)-u(t)\right\rangle d t \geq 0 .
\end{aligned}
$$

By lemma 2.3,

$$
\begin{aligned}
& \int_{0}^{T} e^{-k t}\left\langle C\left(e^{k t} \bar{v}(t)\right)-C\left(e^{k t} \bar{u}(t)\right), \bar{v}(t)-\bar{u}(t)\right\rangle d t \\
& =\int_{0}^{T} e^{-2 k t}\langle C(v(t))-C(u(t)), v(t)-u(t)\rangle d t \leq \frac{C}{\sqrt{2 k}}\|\bar{v}-\bar{u}\|_{X}^{2} .
\end{aligned}
$$

Choosing $k$ so that $k>\mu_{0}$ and $\frac{C}{\sqrt{2 k}}<\frac{\mu_{0}}{2}$, we obtain the following estimate:

$$
\int_{0}^{T}\left\langle V_{k}(\bar{v}(t))-V_{k}(\bar{u}(t)), \bar{v}(t)-\bar{u}(t)\right\rangle d t \geq \frac{\mu_{0}}{2}\|\bar{v}-\bar{u}\|_{X}^{2} .
$$

Hence, the operator $V_{k}$ is monotone. 
Note that $V_{k}(0)=0$. To prove the coercivity property of the operator $V_{k}$ we substitute $\bar{u}=0$ into (2.14) to obtain

$$
\int_{0}^{T}\left\langle V_{k}(\bar{v}(t)), \bar{v}(t)\right\rangle d t \geq \frac{\mu_{0}}{2}\|\bar{v}\|_{X}^{2} .
$$

Using the above propositions we will show that the map $\mathcal{A}$ is invertible and expanding with respect to certain special norms.

Theorem 2.1. Let functions $\mu_{i}(s), i=1,2$ satisfy assumptions $\left.M_{1}\right)$ $\left.M_{2}\right)$. Then the map $\mathcal{A}: W \rightarrow X^{*} \times H$ is invertible and for all functions $v, u \in W$ we have the following estimate:

$$
\|v-u\|_{k, X C} \leq C\|\mathcal{A}(v)-\mathcal{A}(u)\|_{k, X^{*} \times H},
$$

for $k$ large enough, where the constant $C$ is independent of $u, v$ and the choice of $k$.

Proof. 1) Let us show the invertibility of the map $\mathcal{A}$. For this purpose it is sufficient to show that the operator equation

$$
\mathcal{A}(v)=(\varphi, a)
$$

has a unique solution for each pair $\varphi \in X^{*}$ and $a \in H$.

The operator equation (2.16) is equivalent to the problem (2.11) and, hence, the problem (2.12). We can rewrite the problem (2.12) as follows:

$$
\bar{v}^{\prime}+V_{k}(\bar{v})=\varphi, \quad \bar{v}(0)=a .
$$

Since the operator $V_{k}$ is a continuous monotone coercive Volterra operator (according to the terminology of $[6]$ ), this problem has a unique solution by [6, Theorem 1.1]. The map $a \mapsto v$ is continuous from $H$ to $C([0, T], H)$. It follows that the map $\mathcal{A}$ is invertible.

2) Let $u, v \in W$. We shall now prove the estimate (2.15). Set $\mathcal{A}(v)=$ $\left(\varphi, v^{0}\right), \mathcal{A}(u)=\left(\psi, u^{0}\right)$. Then

$$
\begin{array}{ll}
v^{\prime}+\mu_{0} A v+B_{1}(v)+C(v)=\varphi, & v(0)=v^{0}, \\
u^{\prime}+\mu_{0} A u+B_{1}(u)+C(u)=\psi, & u(0)=u^{0} .
\end{array}
$$

Letting

$$
v(t)=e^{k t} \bar{v}(t), \quad u(t)=e^{k t} \bar{u}(t), \quad \varphi(t)=e^{k t} \bar{\varphi}(t), \quad \psi(t)=e^{k t} \bar{\psi}(t)
$$

in the above equations and then multiplying the equations by $e^{-k t}$, we obtain

$$
\begin{aligned}
& \bar{v}^{\prime}+k \bar{v}+\mu_{0} A \bar{v}+e^{-k t} B_{1}\left(e^{k t} \bar{v}(t)\right)+e^{-k t} C\left(e^{k t} \bar{v}(t)\right)=\bar{\varphi}, \quad \bar{v}(0)=v^{0}, \\
& \bar{u}^{\prime}+k \bar{u}+\mu_{0} A \bar{u}+e^{-k t} B_{1}\left(e^{k t} \bar{u}(t)\right)+e^{-k t} C\left(e^{k t} \bar{u}(t)\right)=\bar{\psi}, \quad \bar{u}(0)=u^{0} .
\end{aligned}
$$


Subtract the second equality from the first one and consider the actions of the relevant functionals on the function $\bar{v}(t)-\bar{u}(t)$ :

$$
\begin{aligned}
& \frac{1}{2} \frac{d}{d t}\|\bar{v}(t)-\bar{u}(t)\|_{H}^{2}+k\|\bar{v}(t)-\bar{u}(t)\|_{H}^{2} \\
& \quad+\mu_{0}((\bar{v}(t)-\bar{u}(t), \bar{v}(t)-\bar{u}(t)) \\
& \quad+e^{-k t}\left\langle B_{1}\left(e^{k t} \bar{v}(t)\right)-B_{1}\left(e^{k t} \bar{u}(t)\right), \bar{v}(t)-\bar{u}(t)\right\rangle \\
& \quad+e^{-k t}\left\langle C\left(e^{k t} \bar{v}(t)\right)-C\left(e^{k t} \bar{u}(t)\right), \bar{v}(t)-\bar{u}(t)\right\rangle \\
& =\langle\bar{\varphi}(t)-\bar{\psi}(t), \bar{v}(t)-\bar{u}(t)\rangle .
\end{aligned}
$$

Integrating both sides of this equality from 0 to $t$ and using $(2.9),(2.13)$, we obtain

$$
\begin{gathered}
\frac{1}{2}\|\bar{v}(t)-\bar{u}(t)\|_{H}^{2}-\frac{1}{2}\left\|v^{0}-u^{0}\right\|_{H}^{2}+k\|\bar{v}-\bar{u}\|_{L^{2}\left(Q_{t}\right)}^{2} \\
+\mu_{0} \sum_{i=1}^{n}\left\|\frac{\partial \bar{v}}{\partial x_{i}}-\frac{\partial \bar{u}}{\partial x_{i}}\right\|_{L^{2}\left(Q_{t}\right)}^{2} \leq \frac{C}{\sqrt{2 k}}\|\bar{v}-\bar{u}\|_{X}^{2} \\
+\int_{0}^{T}\|\bar{\varphi}(t)-\bar{\psi}(t)\|_{V^{*}}\|\bar{v}(t)-\bar{u}(t)\|_{H} d t,
\end{gathered}
$$

where $Q_{t}=(0, t) \times \Omega$. It follows that for $k>\mu_{0}$ we have

$$
\begin{aligned}
& \max _{0 \leq t \leq T}\|\bar{v}(t)-\bar{u}(t)\|_{H}^{2}+2 \mu_{0} \sum_{i=1}^{n}\left\|\frac{\partial \bar{v}}{\partial x_{i}}-\frac{\partial \bar{u}}{\partial x_{i}}\right\|_{L^{2}\left(Q_{T}\right)}^{2} \\
& \leq\left\|v^{0}-u^{0}\right\|_{H}^{2}+\frac{2 C}{\sqrt{2 k}}\|\bar{v}-\bar{u}\|_{X}^{2}+2 \int_{0}^{T}\|\bar{\varphi}(t)-\bar{\psi}(t)\|_{V^{*}}\|\bar{v}(t)-\bar{u}(t)\|_{H} d t .
\end{aligned}
$$

By the Cauchy inequality for $\varepsilon>0$,

$$
\begin{aligned}
& \int_{0}^{T}\|\bar{\varphi}(t)-\bar{\psi}(t)\|_{V^{*}} \cdot\|\bar{v}(t)-\bar{u}(t)\|_{H} d t \\
& \leq \frac{\varepsilon}{2} \int_{0}^{T}\|\bar{v}(t)-\bar{u}(t)\|_{H}^{2} d t+\frac{1}{2 \varepsilon} \int_{0}^{T}\|\bar{\varphi}(t)-\bar{\psi}(t)\|_{V^{*}}^{2} d t \\
& \leq \frac{\varepsilon}{2}\|\bar{v}-\bar{u}\|_{X}^{2}+\frac{1}{2 \varepsilon}\|\bar{\varphi}-\bar{\psi}\|_{X^{*}}^{2} .
\end{aligned}
$$

Hence,

$$
\begin{aligned}
& \max _{0 \leq t \leq T}\|\bar{v}(t)-\bar{u}(t)\|_{H}^{2}+2 \mu_{0} \sum_{i=1}^{n}\left\|\frac{\partial \bar{v}}{\partial x_{i}}-\frac{\partial \bar{u}}{\partial x_{i}}\right\|_{L^{2}\left(Q_{T}\right)}^{2} \\
& \leq\left(\frac{\sqrt{2} C}{\sqrt{k}}+\varepsilon\right)\|\bar{v}-\bar{u}\|_{X}^{2}+\left\|v^{0}-u^{0}\right\|_{H}^{2}+\frac{1}{\varepsilon}\|\bar{\varphi}-\bar{\psi}\|_{X^{*}}^{2} .
\end{aligned}
$$


Since $\|\bar{v}-\bar{u}\|_{X}^{2} \leq C_{1}\|\bar{v}-\bar{u}\|_{X C}^{2}$ for some $C_{1}>0$, the above inequality implies

$$
\begin{aligned}
\|\bar{v}-\bar{u}\|_{X C}^{2} \leq & \frac{2}{\min \left\{1,2 \mu_{0}\right\}} C_{1}\left(\frac{\sqrt{2} C}{\sqrt{k}}+\varepsilon\right)\|\bar{v}-\bar{u}\|_{X C}^{2} \\
& +\frac{2}{\min \left\{1,2 \mu_{0}\right\}}\left(\left\|v^{0}-u^{0}\right\|_{H}^{2}+\frac{1}{\varepsilon}\|\bar{\varphi}-\bar{\psi}\|_{X^{*}}^{2}\right) .
\end{aligned}
$$

Choosing $k$ large enough and $\varepsilon$ small enough, so that

$$
\frac{2}{\min \left\{1,2 \mu_{0}\right\}} C_{1}\left(\frac{\sqrt{2} C}{\sqrt{k}}+\varepsilon\right)<\frac{1}{2},
$$

we get the estimate:

$$
\|\bar{v}-\bar{u}\|_{X C}^{2} \leq \frac{4}{\min \left\{1,2 \mu_{0}\right\}}\left(\left\|v^{0}-u^{0}\right\|_{H}^{2}+\frac{1}{\varepsilon}\|\bar{\varphi}-\bar{\psi}\|_{X^{*}}^{2}\right) .
$$

Hence, $\|\bar{v}-\bar{u}\|_{X C} \leq C\left(\left\|v^{0}-u^{0}\right\|_{H}^{2}+\|\bar{\varphi}-\bar{\psi}\|_{X^{*}}^{2}\right)$, for some constant $C$. Since $\|\bar{v}-\bar{u}\|_{X C}=\|v-u\|_{k, X C},\|\bar{\varphi}-\bar{\psi}\|_{X^{*}}=\|\varphi-\psi\|_{k, X^{*}}$ and $\| \mathcal{A}(v)-$ $\mathcal{A}(u)\left\|_{k, X^{*} \times H}=\right\| \varphi-\psi\left\|_{k, X^{*}}+\right\| v^{0}-u^{0} \|_{H}$, the last inequality may be rewritten in the form (2.15).

\subsection{Properties of the operator $K_{\varepsilon}$.}

Theorem 2.2. For each $\varepsilon>0$ the maps

$$
K_{\varepsilon}: W \rightarrow X^{*} \times H \text { and } D_{\varepsilon}: W \rightarrow X^{*}
$$

are completely continuous.

Proof. By definition, $K_{\varepsilon}(v)=\left(D_{\varepsilon}(v), 0\right)$. Thus, it is sufficient to show that the operator $D_{\varepsilon}$ is completely continuous.

The operator $D_{\varepsilon}$ is defined by

$$
\left\langle D_{\varepsilon}(v), h\right\rangle=\sum_{i, j=1}^{n} \int_{\Omega} \frac{v_{i} v_{j}}{1+\varepsilon|v|^{2}} \cdot \frac{\partial h_{j}}{\partial x_{i}} d x \quad \text { for } \quad v, h \in V .
$$

Therefore, the operator $D_{\varepsilon}$ is completely continuous if each one of the operators

$$
d_{i j}: W \subset L^{2}\left(Q_{T}\right) \rightarrow L^{2}\left(Q_{T}\right), \quad d_{i j}(v)=\frac{v_{i} v_{j}}{1+\varepsilon|v|^{2}}, \quad i, j=\overline{1, n}
$$

is completely continuous. By Sobolev's embedding theorem [15, Theorem 1.1], the embedding $V \subset L^{2}(\Omega)$ is completely continuous. Hence, the embedding $W \subset L^{2}\left(Q_{T}\right)$ is completely continuous by theorem 2.1 ([15], p. 217). The estimate $\left|d_{i j}(v)\right|<\frac{1}{\varepsilon}$ implies that the Nemytskii operator $d_{i j}$ : $L^{2}\left(Q_{T}\right) \rightarrow L^{2}\left(Q_{T}\right)$ is continuous. Hence, the operator

$$
d_{i j}: W \subset L^{2}\left(Q_{T}\right) \rightarrow L^{2}\left(Q_{T}\right)
$$

is completely continuous as a composition of the completely continuous embedding $W \subset L^{2}\left(Q_{T}\right)$ and the continuous map $d_{i j}: L^{2}\left(Q_{T}\right) \rightarrow L^{2}\left(Q_{T}\right)$. 
2.5. Properties of the map $\boldsymbol{g}$. In this subsection we show that the map $g$ is $\mathcal{A}$-condensing with respect to the Kuratovskii's measure of noncompactness $\gamma_{k}$.

We recall some necessary notions (see [3]).

Definition 2.1. The nonnegative function $\psi$ is called a measure of noncompactness in a Banach space $E$, for every bounded subset $M$ in $E$ there exists a number $\psi(M)$ such that:

1) $\psi(\overline{c o} M)=\psi(M)$, where $\overline{c o} M$ is the convex hull of the set $M$;

2) $M_{1} \subset M_{2}$ implies $\psi\left(M_{1}\right) \leq \psi\left(M_{2}\right)$.

In this work we shall use Kuratovskii's measure of noncompactness in spaces $W$ and $X^{*} \times H$.

Definition 2.2. The number $\gamma_{k}(M)$ is called Kuratovskii's measure of noncompactness of the set $M \subset W$ if it is equal to the infimum of all numbers $d>0$ for which $M$ may be represented as a union of a finite number of subsets $M_{i}$ with diameters less thand. We mean here a diameter with respect to the norm $\|\cdot\|_{k, X}$.

In the same way we define the measure of noncompactness $\gamma_{k}$ in the space $X^{*} \times H$. Kuratovskii's measure of noncompactness in the space $X^{*} \times H$ has the following properties:

1) $\bar{M}$ is compact iff $\gamma_{k}(M)=0$;

2) $\gamma_{k}\left(M_{1} \cup M_{2}\right)=\max \left\{\gamma_{k}\left(M_{1}\right), \gamma_{k}\left(M_{2}\right)\right\}$;

3) $\gamma_{k}\left(c_{1} M_{1}+c_{2} M_{2}\right) \leq\left|c_{1}\right| \gamma_{k}\left(M_{1}\right)+\left|c_{2}\right| \gamma_{k}\left(M_{2}\right)$ for all numbers $c_{1}, c_{2}$ and subsets $M_{1}, M_{2}$ in $X^{*} \times H$.

Definition 2.3. The map $g$ on $W$ is said to be $\mathcal{A}$-condensing with respect to the measure of noncompactness $\gamma_{k}$ on $X^{*} \times H$ if

$$
\gamma_{k}(g(M))<\gamma_{k}(\mathcal{A}(M))
$$

for every subset $M$ of $W$ such that sets $g(M)$ and $\mathcal{A}(M)$ are bounded and $\gamma_{k}(g(M)) \neq 0$.

The next lemma is an auxiliary one.

Lemma 2.5. If a matrix-function $a(t, s, x, v, w)$ satisfies the conditions $\left.\left.A_{1}\right)-A_{3}\right)$, then for all functions $u, v, w \in L^{2}\left(Q_{T}\right)$ the following estimate is valid:

$$
\begin{aligned}
& \| \int_{0}^{t} \mid a(t, s, x, u(s), v(s))-a\left(t, s, x, u(s), w(s) \mid d s \|_{k, L^{2}\left(Q_{T}\right)}\right. \\
& \leq C \sqrt{\frac{T}{2 k}}\left\|\mathcal{L}_{2}\right\|_{L^{\infty}(Q d)}\|v-w\|_{k, L^{2}\left(Q_{T}\right)}
\end{aligned}
$$

with $C$ independent of $u, v, w$ and $k>0$. 
Proof. Let $\bar{v}(t)=e^{-k t} v(t), \bar{w}(t)=e^{-k t} w(t)$. From the assumption $\left.A_{3}\right)$ it follows that

$$
\begin{aligned}
& e^{-k t} \int_{0}^{t}|a(t, s, x, u(s), v(s))-a(t, s, x, u(s), w(s))| d s \\
& \leq e^{-k t} \int_{0}^{t} \mathcal{L}_{2}(t, s, x)|v(s)-w(s)| d s \\
& \leq\left\|\mathcal{L}_{2}\right\|_{L^{\infty}(Q d)} \int_{0}^{t} e^{-k(t-s)}|\bar{v}(s)-\bar{w}(s)| d s .
\end{aligned}
$$

Then

$$
\begin{aligned}
& \left\|\int_{0}^{t}|a(t, s, x, u(s), v(s))-a(t, s, x, u(s), w(s))| d s\right\|_{k, L^{2}\left(Q_{T}\right)} \\
& =\left\|e^{-k t} \int_{0}^{t}|a(t, s, x, u(s), v(s))-a(t, s, x, u(s), w(s))| d s\right\|_{L^{2}\left(Q_{T}\right)} \\
& \leq\left\|\mathcal{L}_{2}\right\|_{L^{\infty}(Q d)}\left\|\int_{0}^{t} e^{-k(t-s)}|\bar{v}(s)-\bar{w}(s)| d s\right\|_{L^{2}\left(Q_{T}\right)} \\
& =\left\|\mathcal{L}_{2}\right\|_{L^{\infty}(Q d)} \cdot\left(\int_{0}^{T} \int_{\Omega}\left(\int_{0}^{t} e^{-k(t-s)}|\bar{v}(s)-\bar{w}(s)| d s\right)^{2} d x d t\right)^{1 / 2}
\end{aligned}
$$

and, by Hölder's inequality,

$$
\begin{aligned}
& \leq\left\|\mathcal{L}_{2}\right\|_{L^{\infty}(Q d)} \cdot\left(\int_{0}^{T}\left(\int_{0}^{t} e^{-2 k(t-s)} d s\right)\left(\int_{0}^{t} \int_{\Omega}|\bar{v}(s)-\bar{w}(s)|^{2} d x d s\right) d t\right)^{1 / 2} \\
& \leq\left\|\mathcal{L}_{2}\right\|_{L^{\infty}(Q d)} \cdot\|\bar{v}-\bar{w}\|_{L^{2}\left(Q_{T}\right)} \cdot\left(\int_{0}^{T} \int_{0}^{t} e^{-2 k(t-s)} d s d t\right)^{1 / 2} .
\end{aligned}
$$

The required estimate (2.17) follows from the equality (2.10) and from the relation $\|\bar{v}-\bar{w}\|_{L^{2}\left(Q_{T}\right)}=\|v-w\|_{k, L^{2}\left(Q_{T}\right)}$.

Theorem 2.3. If a matrix-function $a(t, s, x, v, w)$ satisfies the assumptions $\left.\left.A_{1}\right)-A_{3}\right)$, then the map $g: W \rightarrow X^{*} \times H$ is $\mathcal{A}$-condensing with respect to Kuratovskii's measure of noncompactness $\gamma_{k}$ for all sufficiently large $k$.

Proof. We define an auxiliary map $p: L^{2}\left(Q_{T}\right) \times X \rightarrow X^{*}$ by the equality

$$
\langle p(v, w), h\rangle=-\int_{\Omega} \int_{0}^{t} a\left(t, s, x, v(s, x), D^{1} w(s, x)\right) d s: D^{1} h(x) d x,
$$


for $h \in V$. The continuity of the map $p$ is proved in the same way as the continuity of $Q$ in lemma 2.1. Moreover, $g(v)=(p(v, v), 0)$ for $v \in X$.

Let $M \subset W$ be an arbitrary bounded set. As we mentioned above, the embedding $W \subset L^{2}\left(Q_{T}\right)$ is completely continuous. Hence, the set $M$ is completely bounded in $L^{2}\left(Q_{T}\right)$. Then for every $w \in X$ the set $p(M, w)$ is completely bounded. By lemma 2.5 the map $p(v, w)$ is Lipschitz continuous in its second variable with Lipschitz constant $\sqrt{\frac{T}{2 k}}\left\|\mathcal{L}_{2}\right\|_{L^{\infty}(Q d)}$. Hence, by $\left[3\right.$, Theorem 1.5.7], the map $g$ is $\sqrt{\frac{T}{2 k}}\left\|\mathcal{L}_{2}\right\|_{L^{\infty}(Q d)}$-bounded with respect to Hausdorff's measure of noncompactness $\chi_{k}$, i.e.

$$
\chi_{k}(g(M)) \leq \sqrt{\frac{T}{2 k}}\left\|\mathcal{L}_{2}\right\|_{L^{\infty}(Q d)} \chi_{k}(M) .
$$

Here, $\chi_{k}(M)$ is the Hausdorff's measure of noncompactness in the space $X$ with the norm $\|v\|_{k, X}$, and $\chi_{k}(g(M))$ is the Hausdorff's measure of noncompactness in the space $X^{*}$ with the norm $\|f\|_{k, X^{*}}$ (these definitions may be found in [3]).

It is known [3, Theorem 1.1.7] that the Kuratovskii and Hausdorff measures of noncompactness satisfy the following inequality

$$
\chi_{k}(M) \leq \gamma_{k}(M) \leq 2 \chi_{k}(M)
$$

Hence,

$$
\gamma_{k}(g(M)) \leq \sqrt{\frac{2 T}{k}}\left\|\mathcal{L}_{2}\right\|_{L^{\infty}(Q d)} \gamma_{k}(M) .
$$

From (2.15) it follows that after the action of the map $\mathcal{A}$ diameter of the set becomes at least $C$ times less. Hence,

$$
\gamma_{k}(M) \leq C \cdot \gamma_{k}(\mathcal{A}(M)),
$$

with the constant $C$ from estimate $(2.15)$. Here, $\gamma_{k}(\mathcal{A}(M))$ is the Kuratovskii measure of noncompactness in the space $X^{*} \times H$ with the norm $\|f\|_{k, X^{*}}+\|a\|_{H}$, for $(f, a) \in X^{*} \times H$. As $g(M) \subset X^{*} \times\{0\}$, the measures of noncompactness $\gamma_{k}(g(M))$ in the space $X^{*}$ and in the space $X^{*} \times H$ coincide. Thus, from (2.18) and (2.19) we obtain

$$
\gamma_{k}(g(M)) \leq \sqrt{\frac{2 T}{k}}\left\|\mathcal{L}_{2}\right\|_{L^{\infty}(Q d)} C \cdot \gamma_{k}(\mathcal{A}(M)) .
$$

Choosing $k$ large enough so that $\sqrt{\frac{2 T}{k}}\left\|\mathcal{L}_{2}\right\|_{L^{\infty}(Q d)} \cdot C<1$, we obtain the inequality

$$
\gamma_{k}(g(M))<\gamma_{k}(\mathcal{A}(M)) .
$$

The choice of $k$ is independent of the set $M$. 


\section{Estimates of SOlutions And solvability of APPROXimating OPERATOR EQUATIONS}

In this section we establish a priori estimates of solutions of approximating operator equations $\left(2.6_{\varepsilon}\right)$ and prove their solvability using the degree theory for $\mathcal{A}$-condensing perturbations of the map $\mathcal{A}$.

3.1. Estimates of solutions. Given $\varepsilon>0$, consider the auxiliary family of operator equations

$$
\mathcal{A}(v)-\tau K_{\varepsilon}(v)-\tau g(v)=\left(f, v^{0}\right), \quad \tau \in[0,1] .
$$

If $\tau=1$ this equation coincides with $\left(2.6_{\varepsilon}\right)$. If $\tau=0$ we get the equation

$$
\mathcal{A}(v)=\left(f, v^{0}\right)
$$

which has a unique solution.

Theorem 3.1. Suppose that the assumptions $\left.\left.\left.M_{1}\right)-M_{2}\right), A_{1}\right)-A_{3}$ ) are fulfilled. Then every solution $v \in W$ of equation $\left(3.1_{\tau}\right)$ for $\tau \in[0,1]$ satisfies the following estimates:

$$
\begin{gathered}
\|v\|_{X C} \leq C\left(1+\|f\|_{X^{*}}+\left\|v^{0}\right\|_{H}\right), \\
\left\|v^{\prime}\right\|_{L^{2}\left((0, T), V^{*}\right)} \leq C\left(1+\|f\|_{X^{*}}+\left\|v^{0}\right\|_{H}\right),
\end{gathered}
$$

with the constant $C$ depending only on $T, n, \Omega, \varepsilon$, and on constants from the assumptions $\left.\left.\left.M_{1}\right)-M_{2}\right), A_{1}\right)-A_{3}$ ).

Proof. Let $v \in W$ be a solution of equation $\left(3.1_{\tau}\right)$ for some $\tau \in[0,1]$. Since $\mathcal{A}(0)=0$, the estimate (2.15) implies

$$
\|v\|_{k, X C} \leq C\|\mathcal{A}(v)\|_{k, X^{*} \times H}
$$

The function $v$ is a solution of equation $\left(3.1_{\tau}\right)$ and, hence,

$$
\mathcal{A}(v)=\tau K_{\varepsilon}(v)+\tau g(v)+\left(f, v^{0}\right),
$$

provided that

$$
\|v\|_{k, X C} \leq C\left(\left\|D_{\varepsilon}(v)\right\|_{k, X^{*}}+\|Q(v)\|_{k, X^{*}}+\|f\|_{k, X^{*}}+\left\|v^{0}\right\|_{H} .\right.
$$

Evaluate the first and second terms at the right-hand side of the inequality.

Note that, by (2.5),

$$
\left\|D_{\varepsilon}(v)\right\|_{k, X^{*}} \leq\left\|D_{\varepsilon}(v)\right\|_{X^{*}} \leq \frac{C_{0}}{\varepsilon},
$$

where the constant $C_{0}$ is independent of $k$ and $\varepsilon$. 
Let $\bar{v}(t)=e^{-k t} v(t)$. Evaluate the second term. By the definition of $g$ and conditions $\left.\left.A_{2}\right)-A_{3}\right)$,

$$
\begin{aligned}
& \|Q(v)\|_{k, X^{*}} \leq\left\|\int_{0}^{t}\left|a\left(t, s, x, v(s, x), D^{1} v(s, x)\right)\right| d s\right\|_{k, L^{2}\left(Q_{T}\right)} \\
& =\left\|e^{-k t} \int_{0}^{t}\left|a\left(t, s, x, v(s, x), D^{1} v(s, x)\right)\right| d s\right\|_{L^{2}\left(Q_{T}\right)} \\
& \leq\left\|e^{-k t} \int_{0}^{t}\left(\mathcal{L}_{1}(t, s, x)+\mathcal{L}_{2}(t, s, x)\left(|v(s, x)|+\left|D^{1} v(s, x)\right|\right)\right) d s\right\|_{L^{2}\left(Q_{T}\right)} \\
& \leq T\left\|\mathcal{L}_{1}\right\|_{L^{2}(Q d)}+\left\|\mathcal{L}_{2}\right\|_{L^{\infty}(Q d)}\left\|\int_{0}^{t} e^{-k t}\left(|v(s, x)|+\left|D^{1} v(s, x)\right|\right) d s\right\|_{L^{2}\left(Q_{t}\right)} .
\end{aligned}
$$

Repeating the arguments of the proof of lemma 2.5, we obtain

$$
\begin{aligned}
& \|Q(v)\|_{k, X^{*}} \\
& \leq C_{1}\left(1+\left(\int_{0}^{T} \int_{\Omega}\left(\int_{0}^{t} e^{-k(t-s)}\left(|\bar{v}(s, x)|+\left|D^{1} \bar{v}(s, x)\right|\right) d s\right)^{2} d x d t\right)^{1 / 2}\right) \\
& \leq C_{1}\left(1+\left(\int_{0}^{T}\left(\int_{0}^{t} e^{-2 k(t-s)} d s\right) \cdot\left(\int_{0}^{t} \int_{\Omega}\left(|\bar{v}(s, x)|^{2}+\left|D^{1} \bar{v}(s, x)\right|^{2}\right) d x d s\right) d t\right)^{1 / 2}\right) \\
& \leq C_{1}\left(1+\left(\|\bar{v}\|_{L^{2}\left(Q_{T}\right)}+\left\|D^{1} \bar{v}\right\|_{L^{2}\left(Q_{T}\right)}\right) \cdot\left(\int_{0}^{T} \int_{0}^{t} e^{-2 k(t-s)} d s d t\right)^{1 / 2}\right) .
\end{aligned}
$$

From inequality (2.10) and the relation

$$
\|\bar{v}\|_{L^{2}\left(Q_{T}\right)}+\left\|D^{1} \bar{v}\right\|_{L^{2}\left(Q_{T}\right)}=\|v\|_{k, L^{2}\left(Q_{T}\right)}+\left\|D^{1} v\right\|_{k, L^{2}\left(Q_{T}\right)} \leq C\|v\|_{k, X C}
$$

it follows that

$$
\|Q(v)\|_{k, X^{*}} \leq C_{1}\left(\sqrt{\frac{T}{2 k}}\|v\|_{k, X C}+1\right) .
$$

Substituting the estimates of $\left\|D_{\varepsilon}(v)\right\|_{k, X^{*}}$ and $\|Q(v)\|_{k, X^{*}}$ into (3.4), we get

$$
\|v\|_{k, X C} \leq C\left(C_{1}\left(\sqrt{\frac{T}{2 k}}\|v\|_{k, X C}+1\right)+\frac{C_{0}}{\varepsilon}+\|f\|_{k, X^{*}}+\left\|v^{0}\right\|_{H}\right) .
$$

Let $k$ be large enough so that

$$
C \cdot C_{1} \sqrt{\frac{T}{2 k}}<\frac{1}{2}
$$

Then the above inequality may be rewritten as follows:

$$
\|v\|_{k, X C} \leq C\left(1+\|f\|_{k, X^{*}}+\left\|v^{0}\right\|_{H}\right),
$$


with some constant $C$ depending on $\varepsilon$. Taking into account this and the equivalence of norms $\|\cdot\|_{k, X C}$ and $\|\cdot\|_{X C}$, as well as the norms $\|\cdot\|_{k, X^{*}}$ and $\|\cdot\|_{X^{*}}$, we obtain $(3.2)$.

To estimate $\left\|v^{\prime}\right\|_{L^{2}\left((0, T), V^{*}\right)}$, we recall that $v$ is a solution of the equation

$$
v^{\prime}+\mu_{0} A v+B_{1}(v)+C(v)-\tau D_{\varepsilon}(v)-\tau Q(v)=f .
$$

Hence,

$$
\begin{aligned}
\left\|v^{\prime}\right\|_{X^{*}} \leq & \mu_{0}\|A v\|_{X^{*}}+\left\|B_{1}(v)\right\|_{X^{*}}+\|C(v)\|_{X^{*}} \\
& +\left\|D_{\varepsilon}(v)\right\|_{X^{*}}+\|Q(v)\|_{X^{*}}+\|f\|_{X^{*}} .
\end{aligned}
$$

Therefore, from inequalities (2.2), (3.5), we obtain

$$
\left\|v^{\prime}\right\|_{X^{*}} \leq C\left(1+\|v\|_{X}\right)+\frac{C}{\varepsilon}+\|f\|_{X^{*}}
$$

Now estimate (3.3) follows from estimate (3.2).

3.2. The basic facts of the degree theory. We recall some facts about the degree theory for $\mathcal{A}$-condensing perturbations of different classes of maps.

Let $E, F$ be Banach spaces and $D$ a bounded subset of $E$. Consider the set of maps of the following form:

$$
\mathcal{A}-g: \bar{D} \subset E \rightarrow F
$$

where $\mathcal{A}$ is a continuous map, $g$ is continuous and $\mathcal{A}$-condensing with respect to some measure of noncompactness $\psi$ on $F$, and $A(x) \neq g(x)$ for $x \in \partial D$.

Two maps $\mathcal{A}-g_{0}, \mathcal{A}-g_{1}:(\bar{D}, \partial D) \rightarrow(F, F \backslash 0)$, where 0 is the origin of the space $F$, are called "homotopic" if there exists an $\mathcal{A}$-condensing homotopy $g: \bar{D} \times[0,1] \rightarrow F$ such that $g(x, 0)=g_{0}(x), g(x, 1)=g_{1}(x), x \in \bar{D}$ and $\mathcal{A}(x) \neq g(x, t)$, for $x \in \partial D, t \in[0,1]$.

Denote by $[\bar{D}, F, \mathcal{A}]_{Y}$ the set of homotopic classes of maps of the form $\mathcal{A}-g:(\bar{D}, \partial D) \rightarrow(F, F \backslash 0)$, and by $[\bar{D}, F, \mathcal{A}]_{C}$ the set of homotopic classes $\mathcal{A}-K:(\bar{D}, \partial D) \rightarrow(F, F \backslash 0)$, where $K$ is a completely continuous map (the homotopies have the form $\mathcal{A}-K$, where $K: \bar{D} \times[0,1] \rightarrow F$, is a completely continuous).

It is known [4, Theorem 4] that the natural embedding

$$
i_{*}:[\bar{D}, F, \mathcal{A}]_{C} \rightarrow[\bar{D}, F, \mathcal{A}]_{Y}
$$

is a bijection. This means that for every map $\mathcal{A}-g$ there exists a homotopic map $\mathcal{A}-K$. If we have a degree for a map $\mathcal{A}-K$, then we define

$$
\operatorname{deg}(\mathcal{A}-g, \bar{D}, 0)=\operatorname{deg}(\mathcal{A}-K, \bar{D}, 0) .
$$

In this work we consider an invertible map $\mathcal{A}$. The construction of the degree of completely continuous perturbations of the invertible map $\mathcal{A}$ is assumed to be well known.

We recall some properties of the degree for maps of the form $\mathcal{A}-g$, where $\mathcal{A}$ is invertible and $g$ is $\mathcal{A}$-condensing. These properties will be useful in what follows.

1. If $\operatorname{deg}\left(\mathcal{A}-g, \bar{D}, y_{0}\right)=\operatorname{deg}\left(\mathcal{A}-g-y_{0}, \bar{D}, 0\right) \neq 0$, then the equation $\mathcal{A}(x)-g(x)=y_{0}$ has a solution in $D$. 
2. If $\mathcal{A}(x) \neq \operatorname{tg}(x)+y_{0}$ for all $x \in \partial D$ and $t \in[0,1]$, then

$$
\operatorname{deg}\left(\mathcal{A}-g, \bar{D}, y_{0}\right)=\operatorname{deg}\left(\mathcal{A}, \bar{D}, y_{0}\right) .
$$

3. If the equation $\mathcal{A}(x)=y_{0}$ has a solution in $D$ then $\operatorname{deg}\left(\mathcal{A}, \bar{D}, y_{0}\right)=1$.

\subsection{Solvability theorem for $\left(\mathbf{2 . 6}_{\varepsilon}\right)$.}

Theorem 3.2. Let the assumptions $\left.M_{1}\right)-M_{2}$ ) and $\left.\left.A_{1}\right)-A_{3}\right)$ hold. Then for all $f \in X^{*}, v^{0} \in H$ and $\varepsilon>0$ the equation $\left(2.6_{\varepsilon}\right)$ has at least one solution $v \in W$.

Proof. Let $f \in X^{*}, v^{0} \in H$ and $\varepsilon>0$. Note that $\left(2.6_{\varepsilon}\right)$ coincides with the equation $\left(3.1_{1}\right)$. By theorem 3.1, all solutions of the family of equations $\left(3.1_{\tau}\right), \tau \in[0,1]$, are contained in the ball $B_{R} \subset W$ with radius $R=$ $C\left(2+\|f\|_{X^{*}}+\left\|v^{0}\right\|_{H}\right)$ and center at zero, and there are no solutions on the boundary of the ball. Therefore, for each $\tau \in[0,1]$, the degree is defined for the map $\mathcal{A}-\tau\left(K_{\varepsilon}+g\right)$ on the ball $B_{R}: \operatorname{deg}\left(\mathcal{A}-\tau\left(K_{\varepsilon}+g\right), B_{R},\left(f, v^{0}\right)\right)$, where the sum of the completely continuous map $K_{\varepsilon}$ and the $\mathcal{A}$-condensing map $g$ is a $\mathcal{A}$-condensing map.

The map $\mathcal{A}$ is invertible. Hence, from the choice of $R$ it follows that the equation $\mathcal{A}(v)=\left(f, v^{0}\right)$ has a solution in the ball $B_{R}$. Then, by property 3 of the degree,

$$
\operatorname{deg}\left(\mathcal{A}, B_{R},\left(f, v^{0}\right)\right)=1 .
$$

From property 2 we obtain

$$
\operatorname{deg}\left(\mathcal{A}-K_{\varepsilon}-g, B_{R},\left(f, v^{0}\right)\right)=\operatorname{deg}\left(\mathcal{A}, B_{R},\left(f, v^{0}\right)\right)=1 .
$$

Finally, property 1 implies that $\left(3.1_{1}\right)$ has a solution in the ball $B_{R}$.

\section{EXISTENCE THEOREM FOR WEAK SOLUTIONS}

In this section we apply approximate solutions to obtain existence results for weak solutions of the initial-boundary value problem for Navier-Stokestype equations.

4.1. A priori estimates for $\left(\mathbf{2}_{\boldsymbol{\varepsilon}} \mathbf{6}\right)$. Here we obtain a priori estimates of solutions of equations $\left(2.6_{\varepsilon}\right), \varepsilon \geq 0$, which are independent of the parameter $\varepsilon$.

Theorem 4.1. Suppose that the conditions $\left.\left.\left.\left.A_{1}\right)-A_{3}\right), M_{1}\right)-M_{2}\right)$ are fulfilled. Then for every solution $v \in W$ of the equations $\left(2.6_{\varepsilon}\right)$, for $\varepsilon>0$, the following estimate is valid:

$$
\|v\|_{X C} \leq C\left(1+\|f\|_{X^{*}}+\left\|v^{0}\right\|_{H}\right),
$$

with the constant $C$ independent of $\varepsilon$.

Proof. Let $v \in W$ be a solution of the equation $\left(2.6_{\varepsilon}\right)$, for some $\varepsilon>0$. Then

$$
v^{\prime}+\mu_{0} A v-D_{\varepsilon}(v)+B_{1}(v)+C(v)-Q(v)=f .
$$

Consider the action on the element $v$ of the above functionals:

$$
\left\langle v^{\prime}, v\right\rangle+\mu_{0}\langle A v, v\rangle-\left\langle D_{\varepsilon}(v), v\right\rangle+\left\langle B_{1}(v), v\right\rangle+\langle C(v), v\rangle-\langle Q(v), v\rangle=\langle f, v\rangle .
$$


We show that $\left\langle D_{\varepsilon}(v), v\right\rangle=0$. In fact,

$$
\begin{aligned}
\left\langle D_{\varepsilon}(v), v\right\rangle & =\sum_{i, j=1}^{n} \int_{\Omega} \frac{v_{i}}{1+\varepsilon|v|^{2}} \cdot \frac{1}{2} \frac{\partial v_{j}^{2}}{\partial x_{i}} d x \\
& =\sum_{i=1}^{n} \int_{\Omega} v_{i} \frac{\partial \ln \left(1+\varepsilon|v|^{2}\right)}{\partial x_{i}} \cdot \frac{1}{2 \varepsilon} d x \\
& =-\frac{1}{2 \varepsilon} \int_{\Omega} \ln \left(1+\varepsilon|v|^{2}\right) \sum_{i=1}^{n} \frac{\partial v_{i}}{\partial x_{i}} d x=0,
\end{aligned}
$$

by the condition of solenoidality of the function $v$.

Thus, we get

$$
\left\langle v^{\prime}, v\right\rangle+\mu_{0}\langle A v, v\rangle+\left\langle B_{1}(v), v\right\rangle+\langle C(v), v\rangle=\langle\varphi, v\rangle,
$$

where $\varphi=f+Q(v)$. Repeating the arguments of the proof of theorem 2.1 with $u=0, u^{0}=0, \psi=0$, we obtain the estimate

$$
\|v\|_{k, X C} \leq C\left(\|\varphi\|_{k, X^{*}}+\left\|v^{0}\right\|_{H}\right)
$$

for $k$ large enough. Since

$$
\|\varphi\|_{k, X^{*}} \leq\|Q(v)\|_{k, X^{*}}+\|f\|_{k, X^{*}} \leq C_{1}\left(\sqrt{\frac{T}{2 k}}\|v\|_{k, X C}+1\right)+\|f\|_{k, X^{*}}
$$

(by estimate (3.3)), we obtain

$$
\|v\|_{k, X C} \leq C\left(C_{1}\left(\sqrt{\frac{T}{2 k}}\|v\|_{k, X C}+1\right)+\|f\|_{k, X^{*}}+\left\|v^{0}\right\|_{H}\right) .
$$

Choosing $k$ large enough so that $C \cdot C_{1} \cdot \sqrt{\frac{T}{2 k}}<\frac{1}{2}$, we get the estimate

$$
\|v\|_{k, X C} \leq C\left(\|f\|_{k, X^{*}}+\left\|v^{0}\right\|_{H}+1\right),
$$

which is equivalent to (4.1).

Theorem 4.2. Suppose that assumptions $\left.\left.\left.M_{1}\right)-M_{2}\right), A_{1}\right)-A_{3}$ ) are fulfilled. Then every solution $v \in W$ of equation $\left(2.6_{\varepsilon}\right)$ for $\varepsilon>0$ satisfies the following inequality

$$
\left\|v^{\prime}\right\|_{L^{1}\left((0, T), V^{*}\right)} \leq C\left(1+\|f\|_{X^{*}}+\left\|v^{0}\right\|_{H}\right)^{2},
$$

with the constant $C$ independent of $\varepsilon$.

Proof. Repeating the arguments of the proof of theorem 3.1, we obtain the inequality

$$
\begin{aligned}
\left\|v^{\prime}\right\|_{L^{1}\left((0, T), V^{*}\right)} \leq & C\left(\|A v\|_{X^{*}}+\left\|B_{1}(v)\right\|_{X^{*}}+\|C(v)\|_{X^{*}}\right. \\
& \left.+\|Q(v)\|_{X^{*}}+\|f\|_{X^{*}}\right)+\left\|D_{\varepsilon}(v)\right\|_{L^{1}\left((0, T), V^{*}\right)}
\end{aligned}
$$


We evaluate $\left\|D_{\varepsilon}(v)\right\|_{L^{1}\left((0, T), V^{*}\right)}$ as in the proof of lemma 2.1. By definition,

$$
\begin{aligned}
\left\|D_{\varepsilon}(v)\right\|_{V^{*}} & \leq \max _{i, j}\left\|\frac{v_{i} v_{j}}{1+\varepsilon|v|^{2}}\right\|_{L^{2}(\Omega)} \\
& \leq \max _{i, j}\left\|v_{i} v_{j}\right\|_{L^{2}(\Omega)} \leq C\|v\|_{L^{4}(\Omega)}^{2} .
\end{aligned}
$$

Hence,

$$
\left\|D_{\varepsilon}(v)\right\|_{L^{1}\left((0, T), V^{*}\right)} \leq C\|v\|_{L^{2}\left((0, T), L^{4}(\Omega)\right)}^{2} .
$$

Since the embedding $V \subset L^{4}(\Omega)$ is continuous for $n \leq 4$, the embed$\operatorname{ding} X=L^{2}((0, T), V) \subset L^{2}\left((0, T), L^{4}(\Omega)\right)$ is also continuous. Hence, $\|v\|_{L^{2}\left((0, T), L^{4}(\Omega)\right)} \leq C\|v\|_{X}$ and

$$
\left\|D_{\varepsilon}(v)\right\|_{L^{1}\left((0, T), V^{*}\right)} \leq C\|v\|_{X}^{2} .
$$

Using the above estimate, inequalities (2.2) and inequality (4.3), we obtain

$$
\left\|v^{\prime}\right\|_{L^{1}\left((0, T), V^{*}\right)} \leq C\left(\left(1+\|v\|_{X}\right)^{2}+\|f\|_{X^{*}}\right) .
$$

Now, estimate (4.2) follows from estimate (4.1).

\subsection{Existence and uniqueness theorems for a weak solution in the} case $n=2$.

Theorem 4.3. Let $n=2$ and let the assumptions $\left.\left.\left.\left.M_{1}\right)-M_{2}\right), A_{1}\right)-A_{3}\right)$ be fulfilled. Then for every $f \in L^{2}((0, T), H)$ and $v^{0} \in H$ there exists at least one weak solution $v \in W$ of the problem (1.1)-(1.4) satisfying the following inequalities:

$$
\begin{aligned}
& \max _{t \in[0, T]}\|v(t)\|_{H}+\sum_{i=1}^{n}\left\|\frac{\partial v}{\partial x_{i}}\right\|_{L^{2}\left(Q_{T}\right)} \leq C\left(1+\|f\|_{L^{2}((0, T), H)}+\left\|v^{0}\right\|_{H}\right), \\
& \left\|v^{\prime}\right\|_{L^{2}\left((0, T), V^{*}\right)} \leq C\left(1+\|f\|_{L^{2}((0, T), H)}+\left\|v^{0}\right\|_{H}\right)^{2}
\end{aligned}
$$

with $C$ independent of $v, f, v^{0}$.

The next lemma is an auxiliary proposition which is analogous to theorem 4.2 .

Lemma 4.1. Under the conditions of theorem 4.3, every solution $v \in W$ of the operator equation $\left(2.6_{\varepsilon}\right), \varepsilon>0$, satisfies

$$
\left\|v^{\prime}\right\|_{L^{2}\left((0, T), V^{*}\right)} \leq C\left(1+\|f\|_{X^{*}}+\left\|v^{0}\right\|_{H}\right)^{2},
$$

with $C$ independent of $\varepsilon>0$.

Proof. By [15, Lemma 3.3] for $n=2$,

$$
\|v\|_{L^{4}(\Omega)} \leq 2^{1 / 4}\|v\|_{L^{2}(\Omega)}^{1 / 2} \cdot\|\operatorname{grad} v\|_{L^{2}(\Omega)}^{1 / 2} .
$$

From the above and from estimate (4.4) we have

$$
\left\|D_{\varepsilon}(v)\right\|_{V^{*}} \leq C\|v\|_{L^{2}(\Omega)} \cdot\|\operatorname{grad} v\|_{L^{2}(\Omega)} .
$$

Hence,

$$
\left\|D_{\varepsilon}(v)\right\|_{L^{2}\left((0, T), V^{*}\right)} \leq C \max _{t \in[0, T]}\|v(t)\|_{H} \cdot\left\|D^{1} v\right\|_{L^{2}\left(Q_{T}\right)} .
$$


Using the embedding $W \subset C([0, T], H)$ and the definition of norm $\|v\|_{X C}$, we obtain

$$
\max _{t \in[0, T]}\|v(t)\|_{H} \leq\|v\|_{X C} \text { and }\left\|D^{1} v\right\|_{L^{2}\left(Q_{T}\right)} \leq\|v\|_{X C} .
$$

Therefore,

$$
\left\|D_{\varepsilon}(v)\right\|_{L^{2}\left((0, T), V^{*}\right)} \leq C\|v\|_{X C}^{2} .
$$

Hence, repeating the arguments of the proof of theorem 3.1, we get the estimate (4.6).

Proof of theorem 4.3. Choose an arbitrary sequence of positive numbers $\left\{\varepsilon_{m}\right\}$ tending to zero. From theorem 3.2 every equation $\left(2.6_{\varepsilon_{m}}\right)$ has a solution $v^{m}$. We shall show that the sequence $\left\{v^{m}\right\}$ has a convergent subsequence which tends (in the norm of the space $X$ ) to a solution of the problem $(2.3),(2.4)$.

The space $V$ is reflexive and separable, thus the space $X=L^{2}((0, T), V)$ is reflexive [6, Remark 1.11], and its dual space $X^{*}$ is also reflexive [6, Theorem 1.14]. In a reflexive Banach space a bounded closed set is weakly compact. By estimates (4.1), (4.6), the set of solutions $\left\{v^{m}\right\}$ is bounded in $W$. Hence, without loss of generality, passing to subsequences and preserving the notation $\left\{v^{m}\right\}$, we may assume that

$$
\begin{aligned}
& v^{m} \rightarrow v^{*} \text { weakly in } X, \\
& v^{m^{\prime}} \rightarrow v^{*^{\prime}} \text { weakly in } X^{*} .
\end{aligned}
$$

Thus, the function $v^{*}$ belongs to $W$. Using the compactness of the embedding $W \subset L^{2}\left(Q_{T}\right)$, we may assume that

$$
\begin{aligned}
& v^{m} \longrightarrow v^{*} \text { strongly in } L^{2}\left(Q_{T}\right), \\
& v^{m} \longrightarrow v^{*} \text { a. e. in } Q_{T} \text { and } v^{*}(0)=v^{0} .
\end{aligned}
$$

As the space $L^{1}((0, T), H)$ is separable, by $[7$, Theorem 6$]$ we may assume that

$$
v^{m} \rightarrow v^{*} \quad * \text {-weakly in } L^{\infty}((0, T), H) .
$$

Next we show that the sequence $\left\{v^{m}\right\}$ converges to $v^{*}$ with respect to the norm of the space $X$ and that $v^{*}$ is a solution of the problem $(2.3),(2.4)$.

Let $f_{0} \in X^{*}$ be defined by

$$
v^{*^{\prime}}+\mu_{0} A v^{*}-K\left(v^{*}\right)+B_{1}\left(v^{*}\right)+C\left(v^{*}\right)-Q\left(v^{*}\right)=f_{0} .
$$

Since $v^{m}$ is a solution of the equation $\left(2.6_{\varepsilon_{m}}\right)$,

$$
\left(v^{m}\right)^{\prime}+\mu_{0} A v^{m}-D_{\varepsilon_{m}}\left(v^{m}\right)+B_{1}\left(v^{m}\right)+C\left(v^{m}\right)-Q\left(v^{m}\right)=f .
$$

Letting

$$
v^{m}(t)=e^{k t} \bar{v}^{m}(t), \quad v^{*}(t)=e^{k t} \bar{v}^{*}(t), \quad f(t)=e^{k t} \bar{f}(t), \quad f_{0}(t)=e^{k t} \bar{f}_{0}(t)
$$

and multiplying the above equalities by $e^{-k t}$, we obtain

$$
\begin{aligned}
\bar{v}^{m^{\prime}} & +k \bar{v}^{m}+\mu_{0} A \bar{v}^{m}-e^{-k t} D_{e_{m}}\left(e^{k t} \bar{v}^{m}(t)\right)+e^{-k t} B_{1}\left(e^{k t} \bar{v}^{m}(t)\right) \\
& +e^{-k t} C\left(e^{k t} \bar{v}^{m}(t)\right)-e^{-k t} Q\left(e^{k t} \bar{v}^{m}(t)\right)=\bar{f}
\end{aligned}
$$


and

$$
\begin{gathered}
\bar{v}^{*^{\prime}}+k \bar{v}^{*}+\mu_{0} A \bar{v}^{*}-e^{-k t} K\left(e^{k t} \bar{v}^{*}(t)\right)+e^{-k t} B_{1}\left(e^{k t} \bar{v}^{*}(t)\right) \\
+e^{-k t} C\left(e^{k t} \bar{v}^{*}(t)\right)-e^{-k t} Q\left(e^{k t} \bar{v}^{*}(t)\right)=\bar{f}_{0} .
\end{gathered}
$$

We subtract from the first equality the second one and consider action of functionals from both sides of the equality on the function $\bar{v}^{m}(t)-\bar{v}^{*}(t)$ :

$$
\begin{aligned}
& \frac{1}{2} \frac{d}{d t}\left\|\bar{v}^{m}(t)-\bar{v}^{*}(t)\right\|_{H}^{2}+k\left\|\bar{v}^{m}(t)-\bar{v}^{*}(t)\right\|_{H}^{2} \\
& \quad+\mu_{0}\left(\left(\bar{v}^{m}(t)-\bar{v}^{*}(t), \bar{v}^{m}(t)-\bar{v}^{*}(t)\right)\right) \\
& \quad+e^{-k t}\left\langle B_{1}\left(e^{k t} \bar{v}^{m}(t)\right)-B_{1}\left(e^{k t} \bar{v}^{*}(t)\right), \bar{v}^{m}(t)-\bar{v}^{*}(t)\right\rangle \\
& \quad+e^{-k t}\left\langle C\left(e^{k t} \bar{v}^{m}(t)\right)-C\left(e^{k t} \bar{v}^{*}(t)\right), \bar{v}^{m}(t)-\bar{v}^{*}(t)\right\rangle \\
& \quad-e^{-k t}\left\langle D_{\varepsilon_{m}}\left(e^{k t} \bar{v}^{m}(t)\right)-K\left(e^{k t} \bar{v}^{*}(t)\right), \bar{v}^{m}(t)-\bar{v}^{*}(t)\right\rangle \\
& \quad-e^{-k t}\left\langle Q\left(e^{k t} \bar{v}^{m}(t)\right)-Q\left(e^{k t} \bar{v}^{*}(t)\right), \bar{v}^{m}(t)-\bar{v}^{*}(t)\right\rangle \\
&=\left\langle\bar{f}(t)-\bar{f}_{0}(t), \bar{v}^{m}(t)-\bar{v}^{*}(t)\right\rangle .
\end{aligned}
$$

Integrating both sides of the equality in $t$ from 0 to $T$ we get

$$
\begin{aligned}
\frac{1}{2}\left\|\bar{v}^{m}(T)-\bar{v}^{*}(T)\right\|_{H}^{2} & \\
& +k\left\|\bar{v}^{m}-\bar{v}^{*}\right\|_{L^{2}\left(Q_{T}\right)}^{2}+\mu_{0} \sum_{i=1}^{n}\left\|\frac{\partial \bar{v}^{m}}{\partial x_{i}}-\frac{\partial \bar{v}^{*}}{\partial x_{i}}\right\|_{L^{2}\left(Q_{T}\right)}^{2} \\
& +\int_{0}^{T} e^{-2 k t}\left\langle B_{1}\left(e^{k t} \bar{v}^{m}(t)\right)-B_{1}\left(e^{k t} \bar{v}^{*}(t)\right), e^{k t} \bar{v}^{m}(t)-e^{k t} \bar{v}^{*}(t)\right\rangle d t \\
= & -\int_{0}^{T} e^{-2 k t}\left\langle C\left(e^{k t} \bar{v}^{m}(t)\right)-C\left(e^{k t} \bar{v}^{*}(t)\right), e^{k t} \bar{v}^{m}(t)-e^{k t} \bar{v}^{*}(t)\right\rangle d t \\
& +\int_{0}^{T} e^{-2 k t}\left\langle Q\left(e^{k t} \bar{v}^{m}(t)\right)-Q\left(e^{k t} \bar{v}^{*}(t)\right), e^{k t} \bar{v}^{m}(t)-e^{k t} \bar{v}^{*}(t)\right\rangle d t \\
& +\int_{0}^{T} e^{-2 k t}\left\langle D_{\varepsilon_{m}}\left(e^{k t} \bar{v}^{m}(t)\right)-K\left(e^{k t} \bar{v}^{*}(t)\right), e^{k t} \bar{v}^{m}(t)-e^{k t} \bar{v}^{*}(t)\right\rangle d t \\
& +\int_{0}^{T}\left\langle\bar{f}(t)-\bar{f}_{0}(t), \bar{v}^{m}(t)-\bar{v}^{*}(t)\right\rangle d t .
\end{aligned}
$$

Let $k \geq \mu_{0}$, then

$$
k\left\|\bar{v}^{m}-\bar{v}^{*}\right\|_{L^{2}\left(Q_{T}\right)}^{2}+\mu_{0} \sum_{i=1}^{n}\left\|\frac{\partial \bar{v}^{m}}{\partial x_{i}}-\frac{\partial \bar{v}^{*}}{\partial x_{i}}\right\|_{L^{2}\left(Q_{T}\right)}^{2} \geq \mu_{0}\left\|\bar{v}^{m}-\bar{v}^{*}\right\|_{X}^{2} .
$$


Lemma 2.2 implies

$$
\begin{aligned}
& \int_{0}^{T} e^{-2 k t}\left\langle B_{1}\left(e^{k t} \bar{v}^{m}(t)\right)-B_{1}\left(e^{k t} \bar{v}^{*}(t)\right), e^{k t} \bar{v}^{m}(t)-e^{k t} \bar{v}^{*}(t)\right\rangle d t \\
& =\int_{0}^{T} e^{-2 k t}\left\langle B_{1}\left(v^{m}(t)\right)-B_{1}\left(v^{*}(t)\right), v^{m}(t)-v^{*}(t)\right\rangle d t \geq 0 .
\end{aligned}
$$

Hence, the left-hand side of (4.7) is no smaller than $\mu_{0}\left\|\bar{v}^{m}-\bar{v}^{*}\right\|_{X}$. Denote the terms at the right hand side of (4.7) by $I_{1}(m), I_{2}(m), I_{3}(m), I_{4}(m)$, respectively. Then, by (4.7), we obtain the following inequality

$$
\mu_{0}\left\|\bar{v}^{m}-\bar{v}^{*}\right\|_{X}^{2} \leq I_{1}(m)+I_{2}(m)+I_{3}(m)+I_{4}(m) .
$$

Let us estimate each term $I_{i}(m), i=1,2,3,4$.

Estimate for $I_{4}(m)$. As $v^{m} \rightarrow v^{*}$ weakly in $X, \bar{v}^{m} \rightarrow \bar{v}^{*}$ weakly in $X$ too. Then by the definition of weak convergence

$$
I_{4}(m)=\int_{0}^{T}\left\langle\bar{f}(t)-\bar{f}_{0}(t), \bar{v}^{m}(t)-\bar{v}^{*}(t)\right\rangle d t \rightarrow 0 \quad \text { as } m \rightarrow \infty .
$$

Estimate for $I_{1}(m)$. From estimate (2.9)

$$
\begin{aligned}
\left|I_{1}(m)\right| & =\int_{0}^{T} e^{-2 k t}\left\langle C\left(v^{m}(t)\right)-C\left(v^{*}(t)\right), v^{m}(t)-v^{*}(t)\right\rangle d t \\
& \leq \frac{C_{1}}{\sqrt{2 k}}\left\|v^{m}-v^{*}\right\|_{k, X}^{2}=\frac{C_{1}}{\sqrt{2 k}}\left\|\bar{v}^{m}-\bar{v}^{*}\right\|_{X}^{2},
\end{aligned}
$$

with $C_{1}$ independent of $k$ and $m$.

Estimate for $I_{2}(m)$. By definition,

$$
\begin{aligned}
I_{2}(m)= & \int_{0}^{T} e^{-2 k t}\left\langle Q\left(v^{m}(t)\right)-Q\left(v^{*}(t)\right), v^{m}(t)-v^{*}(t)\right\rangle d t \\
= & \int_{0}^{T} e^{-2 k t} \int_{\Omega} \int_{0}^{t}\left(a\left(t, s, x, v^{m}(s, x), D^{1} v^{m}(s, x)\right)\right. \\
& \left.-a\left(t, s, x, v^{*}(s, x), D^{1} v^{*}(s, x)\right)\right) d s: D^{1}\left(v^{m}(t, x)-v^{*}(t, x)\right) d x d t \\
= & \int_{0}^{T} e^{-2 k t} \int_{\Omega} \int_{0}^{t}\left(a\left(t, s, x, v^{m}(s, x), D^{1} v^{m}(s, x)\right)\right. \\
& \left.-a\left(t, s, x, v^{m}(s, x), D^{1} v^{*}(s, x)\right)\right) d s: D^{1}\left(v^{m}(t, x)-v^{*}(t, x)\right) d x d t \\
& +\int_{0}^{T} e^{-2 k t} \int_{\Omega}^{t} \int_{0}^{t}\left(a\left(t, s, x, v^{m}(s, x), D^{1} v^{*}(s, x)\right)\right. \\
& \left.-a\left(t, s, x, v^{*}(s, x), D^{1} v^{*}(s, x)\right)\right) d s: D^{1}\left(v^{m}(t, x)-v^{*}(t, x)\right) d x d t .
\end{aligned}
$$


Denote the terms on the right hand-side of the equality by $I_{2,1}(m)$ and $I_{2,2}(m)$, respectively. We evaluate each one of them. By Hölder's inequality,

$$
\begin{aligned}
& \left|I_{2,1}(m)\right| \\
& \leq \| \int_{0}^{t} \mid a\left(t, s, x, v^{m}(s, x), D^{1} v^{m}(s, x)\right) \\
& \quad-a\left(t, s, x, v^{m}(s, x), D^{1} v^{*}(s, x)\right) \mid d s \|_{k, L^{2}\left(Q_{T}\right)} \\
& \quad \cdot\left\|D^{1}\left(v^{m}(t, x)-v^{*}(t, x)\right)\right\|_{k, L^{2}\left(Q_{T}\right)} .
\end{aligned}
$$

Hence, by inequality (2.17), we get

$$
\begin{aligned}
\left|I_{2,1}(m)\right| & \leq C_{2} \sqrt{\frac{T}{2 k}}\left\|\mathcal{L}_{2}\right\|_{L^{\infty}(Q d)}\left\|v^{m}-v^{*}\right\|_{k, X}^{2} \\
& =C_{2} \sqrt{\frac{T}{2 k}}\left\|\mathcal{L}_{2}\right\|_{L^{\infty}(Q d)}\left\|\bar{v}^{m}-\bar{v}^{*}\right\|_{X}^{2} .
\end{aligned}
$$

We now evaluate the second term $I_{2,2}(m)$. By Hölder's inequality,

$$
\begin{aligned}
\left|I_{2,2}(m)\right| \leq & T \| a\left(t, s, x, v^{m}(s, x), D^{1} v^{*}(s, x)\right) \\
& -a\left(t, s, x, v^{*}(s, x), D^{1} v^{*}(s, x)\right) \|_{L^{2}(Q d)} \\
& \cdot\left\|D^{1}\left(v^{m}-v^{*}\right)\right\|_{L^{2}\left(Q_{T}\right)} .
\end{aligned}
$$

The second factor is bounded. We show that the first factor converges to zero as $m \rightarrow \infty$.

From conditions $\left.\left.A_{1}\right)-A_{3}\right)$ it follows that the Nemytskii operator

$$
a: L^{2}\left(Q_{T}\right) \rightarrow L^{2}(Q d), \quad a(v)=a\left(t, s, x, v(s, x), D^{1} v^{*}(s, x)\right)
$$

is continuous. Since $v^{m} \rightarrow v^{*}$ strongly in $L^{2}\left(Q_{T}\right)$ and $a\left(v^{m}\right) \rightarrow a\left(v^{*}\right)$ strongly in $L^{2}(Q d)$, we have

$$
\begin{aligned}
\left\|a\left(v^{m}\right)-a\left(v^{*}\right)\right\|_{L^{2}(Q d)}= & \| a\left(t, s, x, v^{m}(s, x), D^{1} v^{*}(s, x)\right) \\
& -a\left(t, s, x, v^{*}(s, x), D^{1} v^{*}(s, x)\right) \|_{L^{2}(Q d)} \rightarrow 0
\end{aligned}
$$

as $m \rightarrow \infty$. Hence, $I_{2,2}(m) \rightarrow 0$ for $m \rightarrow \infty$. Thus, we have obtained that

$$
\left|I_{2}(m)\right| \leq C_{2} \sqrt{\frac{T}{2 k}}\left\|\bar{v}^{m}-\bar{v}^{*}\right\|_{X}^{2}+\left|I_{2,2}(m)\right|,
$$

where $I_{2,2}(m) \rightarrow 0$ as $m \rightarrow \infty$. 
Estimate for $I_{3}(m)$. By definition,

$$
\begin{aligned}
I_{3}(m)= & \int_{0}^{T} e^{-2 k t}\left\langle D_{\varepsilon_{m}}\left(v^{m}(t)-K\left(v^{*}(t)\right), v^{m}(t)-v^{*}(t)\right\rangle d t\right. \\
= & \int_{0}^{T} e^{-2 k t}\left\langle D_{\varepsilon_{m}}\left(v^{m}(t)\right), v^{m}(t)\right\rangle d t-\int_{0}^{T} e^{-2 k t}\left\langle K\left(v^{*}(t)\right), v^{*}(t)\right\rangle d t \\
& -\int_{0}^{T} e^{-2 k t}\left\langle K\left(v^{*}(t)\right), v^{m}(t)-v^{*}(t)\right\rangle d t \\
& -\int_{0}^{T} e^{-2 k t}\left\langle D_{\varepsilon_{m}}\left(v^{m}(t)\right)-K\left(v^{*}(t)\right), v^{*}(t)\right\rangle d t .
\end{aligned}
$$

Let us estimate each term on the right-hand side of the above equality. In the proof of theorem 4.1 it was shown that

$$
\left\langle D_{\varepsilon}(v), v\right\rangle=0 \text { for all } v \in V \text { and } \varepsilon>0 .
$$

In the same way we can show that $\langle K(v), v\rangle=0$ for $v \in V$. Therefore,

$$
\int_{0}^{T} e^{-2 k t}\left\langle D_{\varepsilon_{m}}\left(v^{m}(t)\right), v^{m}(t)\right\rangle d t=0
$$

and

$$
\int_{0}^{T} e^{-2 k t}\left\langle K\left(v^{*}(t)\right), v^{*}(t)\right\rangle d t=0 .
$$

Furthermore, by the definition of the weak convergence $v^{m} \rightarrow v^{*}$ in the space $X$,

$$
\int_{0}^{T} e^{-2 k t}\left\langle K\left(v^{*}(t)\right), v^{m}(t)-v^{*}(t)\right\rangle d t \rightarrow 0 \quad \text { as } m \rightarrow \infty .
$$

We show that the last term in the expression $I_{3}(m)$ also converges to zero as $m \rightarrow \infty$. By definition,

$$
\begin{aligned}
& \int_{0}^{T} e^{-2 k t}\left\langle D_{\varepsilon_{m}}\left(v^{m}(t)\right)-K\left(v^{*}(t)\right), v^{*}(t)\right\rangle d t \\
& =\sum_{i, j=1}^{n} \int_{0}^{T} e^{-2 k t} \int_{\Omega}\left(\frac{v_{i}^{m} v_{j}^{m}}{1+\varepsilon_{m}\left|v^{m}\right|^{2}}-v_{i}^{*} v_{j}^{*}\right) \frac{\partial v_{j}^{*}}{\partial x_{i}} d x d t .
\end{aligned}
$$

Since $v^{m} \rightarrow v^{*}$ almost everywhere on $Q_{T}$,

$$
\frac{v_{i}^{m} v_{j}^{m}}{1+\varepsilon_{m}\left|v^{m}\right|^{2}} \rightarrow v_{i}^{*} v_{j}^{*} \text { a. e. on } Q_{T} \text {. }
$$

From the estimate

$$
\|v\|_{L^{4}(\Omega)} \leq 2^{1 / 4}\|v\|_{L^{2}(\Omega)}^{1 / 2} \cdot\|\operatorname{grad} v\|_{L^{2}(\Omega)}^{1 / 2}
$$


for $v \in W$ and $n=2$, it follows that $W \subset L^{4}\left(Q_{T}\right)$, and this embedding is continuous. Therefore,

$$
\left\|\frac{v_{i}^{m} v_{j}^{m}}{1+\varepsilon_{m}\left|v^{m}\right|^{2}}\right\|_{L^{2}\left(Q_{T}\right)} \leq\left\|v_{i}^{m} v_{j}^{m}\right\|_{L^{2}\left(Q_{T}\right)} \leq C\left\|v^{m}\right\|_{L^{4}\left(Q_{T}\right)}^{2} \leq C\left\|v^{m}\right\|_{W}^{2} .
$$

As the set of solutions $\left\{v^{m}\right\}$ is bounded in $W$, the set of functions $\left\{\frac{v_{i}^{m} v_{j}^{m}}{1+\varepsilon_{m}\left|v^{m}\right|^{2}}\right\}$ is bounded in $L^{2}\left(Q_{T}\right)$. Therefore, without loss of generality, we may assume that

$$
\frac{v_{i}^{m} v_{j}^{m}}{1+\varepsilon_{m}\left|v^{m}\right|^{2}} \rightarrow v_{i}^{*} v_{j}^{*} \text { weakly in } L^{2}\left(Q_{T}\right) .
$$

This means that

$$
D_{\varepsilon_{m}}\left(v^{m}\right) \rightarrow K\left(v^{*}\right) \text { weakly in } X^{*},
$$

and, consequently,

$$
\int_{0}^{T} e^{-2 k t}\left\langle D_{\varepsilon_{m}}\left(v^{m}(t)\right)-K\left(v^{*}(t)\right), v^{*}(t)\right\rangle d t \rightarrow 0,
$$

as $m \rightarrow \infty$.

Thus, we have showed that all the terms in the expression for $I_{3}(m)$ are equal to zero or converge to zero as $m \rightarrow \infty$, i.e.,

$$
I_{3}(m) \rightarrow 0 \text { for } m \rightarrow \infty .
$$

Summarizing the above investigation of the terms $I_{i}(m), i=1,2,3,4$, and applying inequalities, we can rewrite (4.8) as follows:

$$
\begin{aligned}
\mu_{0}\left\|\bar{v}^{m}-\bar{v}^{*}\right\|_{X}^{2} \leq & \frac{C_{1}}{\sqrt{2 k}}\left\|\bar{v}^{m}-\bar{v}^{*}\right\|_{X}^{2}+C_{2} \sqrt{\frac{T}{2 k}}\left\|\bar{v}^{m}-\bar{v}^{*}\right\|_{X}^{2} \\
& +\left|I_{2,2}(m)\right|+I_{3}(m)+I_{4}(m) .
\end{aligned}
$$

Choosing sufficiently large $k$ such that $\frac{C_{1}}{\sqrt{2 k}}+C_{2} \sqrt{\frac{T}{2 k}} \leq \frac{\mu_{0}}{2}$, we get

$$
\mu_{0}\left\|\bar{v}^{m}-\bar{v}^{*}\right\|_{X}^{2} \leq 2\left(\left|I_{2,2}(m)\right|+I_{3}(m)+I_{4}(m)\right) .
$$

Each term of the right hand side of the above inequality converges to zero as $m \rightarrow \infty$, and $\left\|\bar{v}^{m}-\bar{v}^{*}\right\|_{X} \rightarrow 0$ as $m \rightarrow \infty$. Hence,

$$
v^{m} \rightarrow v^{*} \text { strongly in } X \text {. }
$$

By lemma 2.1 the maps $A, B_{1}, C, Q$ are continuous on $X$. Hence,

$$
\begin{aligned}
& A v^{m} \rightarrow A v^{*} \text { strongly in } X^{*}, \\
& B_{1}\left(v^{m}\right) \rightarrow B_{1}\left(v^{*}\right) \text { strongly in } X^{*}, \\
& C\left(v^{m}\right) \rightarrow C\left(v^{*}\right) \text { strongly in } X^{*}, \\
& Q\left(v^{m}\right) \rightarrow Q\left(v^{*}\right) \text { strongly in } X^{*} .
\end{aligned}
$$

Passing to the limit, in weak sense, in each term of the equality

$$
\left(v^{m}\right)^{\prime}+\mu_{0} A v^{m}-D_{\varepsilon_{m}}\left(v^{m}\right)+B_{1}\left(v^{m}\right)+C\left(v^{m}\right)-Q\left(v^{m}\right)=f
$$


and applying (4.10), we obtain

$$
\left(v^{*}\right)^{\prime}+\mu_{0} A v^{*}-K\left(v^{*}\right)+B_{1}\left(v^{*}\right)+C\left(v^{*}\right)-Q\left(v^{*}\right)=f .
$$

Thus, we have showed that $v^{*}$ is a solution of equation $(2.3)$ and $v^{*}(0)=v^{0}$.

The uniqueness of weak the solution of the problem (1.1)-(1.4) will be established under assumption that the matrix-function $a(t, s, x, v, w)$ is Lipschitzian in the variables $v, w$, i.e.,

$$
\left.A_{2}^{\prime}\right)\left|a_{i j}(t, s, x, u, v)-a_{i j}(t, s, x, \bar{u}, \bar{v})\right| \leq \mathcal{L}_{2}(t, s, x)(|u-\bar{u}|+|v-\bar{v}|),
$$

for all $(t, s, x) \in Q d, u, \bar{u} \in \mathbb{R}^{n}, v, \bar{v} \in \mathbb{R}^{n^{2}}, i, j \in \overline{1, n}$, where $\mathcal{L}_{2}$ is an essentially bounded function.

Theorem 4.4. Let $n=2$ and conditions $\left.\left.\left.\left.M_{1}\right)-M_{2}\right), A_{1}\right)-A_{2}^{\prime}\right)$ be fulfilled. Then for each $f \in L^{2}((0, T), H), v^{0} \in H$, the weak solution $v \in W$ of the problem $(1.1)-(1.4)$ is unique.

Proof. Suppose, there exists two different solutions $u$ and $v$ of the problem (2.3)-(2.4). Then

$$
\begin{aligned}
& v^{\prime}+\mu_{0} A v-K(v)+B_{1}(v)+C(v)-Q(v)=f \\
& u^{\prime}+\mu_{0} A u-K(u)+B_{1}(u)+C(u)-Q(u)=f .
\end{aligned}
$$

Substituting the following expressions

$$
v(t)=e^{k t} \bar{v}(t), \quad u(t)=e^{k t} \bar{u}(t), \quad f(t)=e^{k t} \bar{f}(t)
$$

in the above equalities, then multiplying them by $e^{-k t}$ and then subtracting the second equality from the first one, we obtain

$$
\begin{aligned}
& (\bar{v}-\bar{u})^{\prime}+k(\bar{u}-\bar{v})+\mu_{0} A(\bar{v}-\bar{u})-e^{-k t}\left(K\left(e^{k t} \bar{v}(t)\right)-K\left(e^{k t} \bar{u}(u)\right)\right) \\
& \quad+e^{-k t}\left(B_{1}\left(e^{k t} \bar{v}(t)\right)-B_{1}\left(e^{k t} \bar{u}(t)\right)+e^{-k t}\left(C\left(e^{k t} \bar{v}(t)\right)-C\left(e^{k t} \bar{u}(t)\right)\right)\right. \\
& \quad-e^{-k t}\left(Q\left(e^{k t} \bar{v}(t)\right)-Q\left(e^{k t} \bar{u}(t)\right)\right) \\
& =0 .
\end{aligned}
$$

Evaluating the functionals from this equality on the function $\bar{v}(t)-\bar{u}(t)$ and then integrating the result in with respect to $t$ from 0 to $\tau$, we arrive at

$$
\begin{aligned}
\frac{1}{2}\|\bar{v}(\tau)-\bar{u}(\tau)\|_{H}^{2}+ & k\|\bar{v}-\bar{u}\|_{L^{2}\left(Q_{\tau}\right)}^{2}+\mu_{0} \sum_{i=1}^{n}\left\|\frac{\partial \bar{v}}{\partial x_{i}}-\frac{\partial \bar{u}}{\partial x_{i}}\right\|_{L^{2}\left(Q_{\tau}\right)}^{2} \\
& +\int_{0}^{\tau} e^{-2 k t}\left\langle B_{1}(v(t))-B_{1}(u(t)), v(t)-u(t)\right\rangle d t \\
= & -\int_{0}^{\tau} e^{-2 k t}\langle C(v(t))-C(u(t)), v(t)-u(t)\rangle d t
\end{aligned}
$$




$$
\begin{aligned}
& +\int_{0}^{\tau} e^{-2 k t}\langle Q(v(t))-Q(u(t)), v(t)-u(t)\rangle d t \\
& +\int_{0}^{\tau} e^{-2 k t}\langle K(v(t))-K(u(t)), v(t)-u(t)\rangle d t .
\end{aligned}
$$

As in the proof of theorem 4.2, we denote the terms on the right-hand side of this equality by $I_{1}(\tau), I_{2}(\tau), I_{3}(\tau)$, respectively, and suppose that $k \geq \mu_{0}$. Then we obtain

$$
\frac{1}{2}\|\bar{v}(\tau)-\bar{u}(\tau)\|_{H}^{2}+\mu_{0}\|\bar{v}-\bar{u}\|_{L^{2}((0, \tau), V)}^{2} \leq I_{1}(\tau)+I_{2}(\tau)+I_{3}(\tau)
$$

Let us estimate the terms $I_{1}(\tau), I_{2}(\tau), I_{3}(\tau)$.

Estimate for $I_{1}(\tau)$. The estimate $(2.9)$ gives

$$
I_{1}(\tau) \leq \frac{C_{1}}{\sqrt{2 k}}\|\bar{v}-\bar{u}\|_{L^{2}((0, \tau), V)}^{2}
$$

with $C_{1}$ independent of $\tau$.

Estimate for $I_{2}(\tau)$. By definition,

$$
\begin{aligned}
I_{2}(\tau)= & \int_{0}^{\tau} e^{-2 k t} \int_{\Omega} \int_{0}^{t}\left(a\left(t, s, x, v(s, x), D^{1} v(s, x)\right)\right. \\
& \left.-a\left(t, s, x, u(s, x), D^{1} u(s, x)\right)\right) d s: D^{1}(v(t, x)-u(t, x)) d x d t .
\end{aligned}
$$

Then, applying Hölder's inequality and (2.10), we have

$$
\begin{aligned}
\left|I_{2}(\tau)\right| \leq & \| \int_{0}^{t} \mid a\left(t, s, x, v(s, x), D^{1} v(s, x)\right)- \\
& -a\left(t, s, x, u(s, x), D^{1} u(s, x)\right) \mid d s \|_{k, L^{2}\left(Q_{\tau}\right)} \\
& \cdot\left\|D^{1}(v(t, x)-u(t, x))\right\|_{k, L^{2}\left(Q_{\tau}\right)} \\
\leq & \left\|\mathcal{L}_{2}\right\|_{L^{\infty}(Q d)} \| \int_{0}^{t}(|v(s, x)-u(s, x)| \\
& \left.+\left|D^{1} v(s, x)-D^{1} u(s, x)\right|\right) d s \|_{k, L^{2}\left(Q_{\tau}\right)} \\
& \cdot\left\|D^{1}(v(t, x)-u(t, x))\right\|_{k, L^{2}\left(Q_{\tau}\right)}
\end{aligned}
$$




$$
\begin{aligned}
= & \left\|\mathcal{L}_{2}\right\|_{L^{\infty}(Q d)} \| \int_{0}^{t} e^{-k(t-s)}(|\bar{v}(s, x)-\bar{u}(s, x)| \\
& \left.+\left|D^{1} \bar{v}(s, x)-D^{1} \bar{u}(s, x)\right|\right) d s \|_{L^{2}\left(Q_{\tau}\right)} \\
& \cdot\left\|D^{1}(\bar{v}(t, x)-\bar{u}(t, x))\right\|_{L^{2}\left(Q_{\tau}\right)} \\
\leq & C\left\|\mathcal{L}_{2}\right\|_{L^{\infty}(Q d)}\left(\int _ { 0 } ^ { \tau } \int _ { \Omega } \left(\int_{0}^{t} e^{-k(t-s)}(|\bar{v}(s, x)-\bar{u}(s, x)|\right.\right. \\
& \left.\left.\left.+\left|D^{1} \bar{v}(s, x)-D^{1} \bar{u}(s, x)\right|\right) d s\right)^{2} d x d t\right)^{1 / 2} \cdot\|\bar{v}-\bar{u}\|_{L^{2}((0, \tau), V)} \\
\leq & C\left\|\mathcal{L}_{2}\right\|_{L^{\infty}(Q d)} \cdot\|\bar{v}-\bar{u}\|_{L^{2}((0, \tau), V)} \\
& \cdot\left(\int _ { 0 } ^ { \tau } ( \int _ { 0 } ^ { t } e ^ { - 2 k ( t - s ) } d s ) \left(\int _ { 0 } ^ { t } \left(|\bar{v}(s, x)-\bar{u}(s, x)|^{2}\right.\right.\right. \\
& \left.\left.\left.\left.+\left|D^{1} \bar{v}(s, x)-D^{1} \bar{u}(s, x)\right|\right)^{2}\right) d x d s\right) d t\right)^{1 / 2} \\
\leq & C\left\|\mathcal{L}_{2}\right\|_{L^{\infty}(Q d)} \cdot \sqrt{\frac{\tau}{2 k}}\|\bar{v}-\bar{u}\|_{L^{2}((0, \tau), V)}^{2},
\end{aligned}
$$

which implies

$$
\left|I_{2}(\tau)\right| \leq \frac{C_{2}}{\sqrt{2 k}}\|\bar{v}-\bar{u}\|_{L^{2}((0, \tau), V)}^{2} .
$$

Estimate for $I_{3}(\tau)$. By definition,

$$
\begin{aligned}
I_{3}(\tau)= & \int_{0}^{\tau} e^{-2 k t} \int_{\Omega} \sum_{i, j=1}^{n}\left(v_{i} v_{j}-u_{i} u_{j}\right) \cdot \frac{\partial\left(v_{j}-u_{j}\right)}{\partial x_{i}} d x d t \\
= & \int_{0}^{\tau} e^{-2 k t} \sum_{i, j=1}^{n} \int_{\Omega}\left(\left(v_{i}-u_{i}\right) v_{j} \cdot \frac{\partial\left(v_{j}-u_{j}\right)}{\partial x_{i}}\right. \\
& \left.+u_{i}\left(v_{j}-u_{j}\right) \frac{\partial\left(v_{j}-u_{j}\right)}{\partial x_{i}}\right) d x d t .
\end{aligned}
$$

Hence, taking into account the condition (1.2), we obtain

$$
\begin{aligned}
I_{3}(\tau) & =-\int_{0}^{\tau} e^{-2 k t} \sum_{i, j=1}^{n} \int_{\Omega}\left(v_{i}-u_{i}\right) \frac{\partial v_{j}}{\partial x_{i}}\left(v_{j}-u_{j}\right) d x d t \\
& =-\int_{0}^{\tau} \sum_{i, j=1}^{n} \int_{\Omega}\left(\bar{v}_{i}-\bar{u}_{i}\right) \frac{\partial v_{j}}{\partial x_{i}}\left(\bar{v}_{j}-\bar{u}_{j}\right) d x d t .
\end{aligned}
$$


From the inequality $\|v\|_{L^{4}(\Omega)} \leq 2^{1 / 4}\|v\|_{L^{2}(\Omega)}^{1 / 2} \cdot\|\operatorname{grad} v\|_{L^{2}(\Omega)}^{1 / 2}([15]$, p. 233$)$, as well as the Schwartz and Hölder inequalities, we obtain

$$
\begin{aligned}
& \left|\sum_{i, j=1}^{n} \int_{\Omega}\left(\bar{v}_{i}-\bar{u}_{i}\right) \frac{\partial v_{j}}{\partial x_{i}}\left(\bar{v}_{j}-\bar{u}_{j}\right) d x\right| \\
& \leq \sum_{i, j=1}^{n}\left\|\bar{v}_{i}-\bar{u}_{i}\right\|_{L^{4}(\Omega)} \cdot\left\|\frac{\partial v_{j}}{\partial x_{i}}\right\|_{L^{2}(\Omega)} \cdot\left\|\bar{v}_{j}-\bar{u}_{j}\right\|_{L^{4}(\Omega)} \\
& \leq\left(\sum_{i=1}^{n}\left\|\bar{v}_{i}-\bar{u}_{i}\right\|_{L^{4}(\Omega)}^{2}\right)^{1 / 2}\left(\sum_{i, j=1}^{n}\left\|\frac{\partial v_{j}}{\partial x_{i}}\right\|_{L^{2}(\Omega)}^{2}\right)^{1 / 2}\left(\sum_{j=1}^{n}\left\|\bar{v}_{j}-\bar{u}_{j}\right\|_{L^{4}(\Omega)}^{2}\right)^{1 / 2} \\
& =\sum_{i=1}^{n}\left\|\bar{v}_{i}-\bar{u}_{i}\right\|_{L^{4}(\Omega)}^{2}\left(\sum_{i, j=1}^{n}\left\|\frac{\partial v_{j}}{\partial x_{i}}\right\|_{L^{2}(\Omega)}^{2}\right)^{1 / 2} \\
& \leq \sqrt{2} \sum_{i=1}^{n}\left(\left\|\bar{v}_{i}-\bar{u}_{i}\right\|_{L^{2}(\Omega)} \cdot\left\|\operatorname{grad}\left(\bar{v}_{i}-\bar{u}_{i}\right)\right\|_{L^{2}(\Omega)}\right)\left(\sum_{j=1}^{n}\left\|\operatorname{grad}\left(v_{j}\right)\right\|_{L^{2}(\Omega)}^{2}\right)^{1 / 2} \\
& \leq \sqrt{2}\left\|\bar{v}^{2}-\bar{u}\right\|_{L^{2}(\Omega)} \cdot\left\|D^{1} \bar{v}-D^{1} \bar{u}\right\|_{L^{2}(\Omega)} \cdot\left\|D^{1} v\right\|_{L^{2}(\Omega)}
\end{aligned}
$$

Thus,

$$
\left|I_{3}(\tau)\right| \leq \sqrt{2} \int_{0}^{\tau}\|\bar{v}(t)-\bar{u}(t)\|_{H} \cdot\left\|D^{1} \bar{v}(t)-D^{1} \bar{u}(t)\right\|_{H} \cdot\left\|D^{1} v(t)\right\|_{H} d t
$$

From the estimates for $I_{1}(\tau)$ and $I_{2}(\tau)$ it follows that

$$
\begin{aligned}
\frac{1}{2}\|\bar{v}(\tau)-\bar{u}(\tau)\|_{H}^{2} & +\mu_{0}\|\bar{v}-\bar{u}\|_{L^{2}((0, \tau), V)}^{2} \\
& \leq \frac{C_{1}}{\sqrt{2 k}}\|\bar{v}-\bar{u}\|_{L^{2}((0, \tau), V)}^{2}+\frac{C_{2}}{\sqrt{2 k}}\|\bar{v}-\bar{u}\|_{L^{2}((0, \tau), V)}^{2}+I_{3}(\tau) .
\end{aligned}
$$

Choosing $k$ large enough, so that $\frac{C_{1}+C_{2}}{\sqrt{2 k}}<\frac{\mu_{0}}{2}$, we obtain

$$
\|\bar{v}(\tau)-\bar{u}(\tau)\|_{H}^{2}+\mu_{0}\|\bar{v}-\bar{u}\|_{L^{2}((0, \tau), V)}^{2} \leq 2 I_{3}(\tau) .
$$

By Cauchy's inequality,

$$
\begin{aligned}
I_{3}(\tau) \leq & \frac{1}{\varepsilon \sqrt{2}} \int_{0}^{\tau}\|\bar{v}(t)-\bar{u}(t)\|_{H}^{2} \cdot\left\|D^{1} v(t)\right\|_{H}^{2} d t \\
& +\frac{\varepsilon}{\sqrt{2}} \int_{0}^{\tau}\left\|D^{1} \bar{v}(t)-D^{1} \bar{u}(t)\right\|_{H}^{2} d t \\
\leq & \frac{1}{\varepsilon \sqrt{2}} \int_{0}^{\tau}\|\bar{v}(t)-\bar{u}(t)\|_{H}^{2} \cdot\left\|D^{1} v(t)\right\|_{H}^{2} d t+\frac{\varepsilon}{\sqrt{2}}\|\bar{v}-\bar{u}\|_{L^{2}((0, \tau), V)}^{2} .
\end{aligned}
$$


Choosing $\varepsilon=\frac{\mu_{0}}{2 \sqrt{2}}$ and substituting the above inequality into (4.12) we get $\|\bar{v}(\tau)-\bar{u}(\tau)\|_{H}^{2}+\frac{\mu_{0}}{2}\|\bar{v}-\bar{u}\|_{L^{2}((0, \tau), v)}^{2} \leq \frac{4}{\mu_{0}} \int_{0}^{\tau}\|\bar{v}(t)-\bar{u}(t)\|_{H}^{2} \cdot\left\|D^{1} v(t)\right\|_{H}^{2} d t$ which gives

$$
\|\bar{v}(\tau)-\bar{u}(\tau)\|_{H}^{2} \leq \frac{4}{\mu_{0}} \int_{0}^{\tau}\|\bar{v}(t)-\bar{u}(t)\|_{H}^{2} \cdot\left\|D^{1} v(t)\right\|_{H}^{2} d t .
$$

Hence, by the Gronwall-Bellman inequality we obtain

$$
\|\bar{v}(\tau)-\bar{u}(\tau)\|_{H}=0 \text { for } \tau \in[0, T]
$$

i.e. $v=u$.

4.3. Existence theorem for weak solutions for $\boldsymbol{n} \leq \mathbf{4}$. In the case $n \leq 4$ we consider the equation (1.1) in the form:

$$
\begin{aligned}
\frac{\partial v}{\partial t}-\mu_{0} \Delta v & +\sum_{i=1}^{n} v_{i} \frac{\partial v}{\partial x_{i}}-\int_{0}^{t} \operatorname{Div}\left(a\left(t, s, x, v(s, x), D^{1} v(s, x)\right) d s\right. \\
& +\operatorname{grad} p=f, \quad(t, x) \in Q_{T},
\end{aligned}
$$

where elements of the matrix-function $a_{i j}$ are defined by

$$
\begin{aligned}
& a_{i j}\left(t, s, x, v(s, x), D^{1} v(s, x)\right) \\
& =b(i, j ; t, s, x): D^{1} v(s, x)+c(i, j ; t, s, x) \cdot v(s, x),
\end{aligned}
$$

and the matrix-functions $b(i, j, \cdot)$ and the vector-function $c(i, j, \cdot)$ are essentially bounded.

In particular, the equation (4.13) contains, as a special case, the equations of the Oldroid mathematical model and its generalizations [2], [8].

The problem of the existence of weak solutions of an initial-boundary value problem for the equation (4.13) is equivalent to the problem

$$
v^{\prime}+\mu_{0} A v-K(v)-\int_{0}^{t} G(t, s, v(s)) d s=f, \quad v(0)=v^{0} .
$$

Moreover, the solution $v \in L^{2}((0, T), V)$ and $v^{\prime} \in L^{1}\left((0, T), V^{*}\right)$.

We first prove an auxiliary proposition.

Lemma 4.2. If sequence $v^{\varepsilon} \in X$ converges weakly in $X$ to $v^{*}$, strongly with respect to the norm $L^{2}((0, T), H)$ and a.e. on $Q_{T}$, then $D_{\varepsilon}\left(v^{\varepsilon}\right)--\rightarrow$ $K\left(v^{*}\right), \varepsilon \rightarrow 0$. The last limit is in the sense of distributions on $[0, T]$ with values in $\mathcal{V}^{*}$.

Proof. By the definition of convergence in the sense of distributions ([6], p. $167)$, it is sufficient to show that

$$
\int_{0}^{T}\left\langle D_{\varepsilon}\left(v^{\varepsilon}\right)-K\left(v^{*}\right), h\right\rangle \psi(t) d t \rightarrow 0
$$


for all $h \in \mathcal{V}$ and for all real-valued functions $\psi$ which are continuously differentiable on $[0, T]$ and have supports in $(0, T)$. Since

$$
\int_{0}^{T}\left\langle D_{\varepsilon}\left(v^{\varepsilon}\right)-K\left(v^{*}\right), h\right\rangle \psi(t) d t=\int_{0}^{T} \int_{\Omega} \sum_{i, j=1}^{n}\left(\frac{v_{i}^{\varepsilon} v_{j}^{\varepsilon}}{1+\varepsilon\left|v^{\varepsilon}\right|^{2}}-v_{i}^{*} v_{j}^{*}\right) \frac{\partial h_{j}}{\partial x_{i}} \psi d x d t,
$$

we have

$$
\begin{aligned}
& \left|\int_{0}^{T}\left\langle D_{\varepsilon}\left(v^{\varepsilon}\right)-K\left(v^{*}\right), h\right\rangle \psi(t) d t\right| \\
& \leq C \max _{i, j}\left\|\frac{v_{i}^{\varepsilon} v_{j}^{\varepsilon}}{1+\varepsilon\left|v^{\varepsilon}\right|^{2}}-v_{i}^{*} v_{j}^{*}\right\|_{L^{1}\left(Q_{T}\right)} \cdot\|h\|_{C^{1}(\bar{\Omega})} \cdot\|\psi\|_{L^{\infty}((0, T), R)} .
\end{aligned}
$$

Consider a chain of inequalities:

$$
\begin{aligned}
& \left\|\frac{v_{i}^{\varepsilon} v_{j}^{\varepsilon}}{1+\varepsilon\left|v^{\varepsilon}\right|^{2}}-v_{i}^{*} v_{j}^{*}\right\|_{L^{1}\left(Q_{T}\right)}+\left\|\frac{\varepsilon\left|v^{\varepsilon}\right|^{2} v_{i}^{*} v_{j}^{*}}{1+\varepsilon\left|v^{\varepsilon}\right|^{2}}\right\|_{L^{1}\left(Q_{T}\right)} \\
& \leq\left\|\frac{v_{i}^{\varepsilon} v_{j}^{\varepsilon}-v_{i}^{*} v_{j}^{*}}{1+\varepsilon\left|v^{\varepsilon}\right|^{2}}\right\|_{L^{1}\left(Q_{T}\right)}+\| \varepsilon\left|v^{\varepsilon}\right|^{2} v_{i}^{*} v_{j}^{*} \\
& \leq\left\|v_{i}^{\varepsilon} v_{j}^{\varepsilon}-v_{i}^{*} v_{j}^{*}\right\|_{L^{1}\left(Q_{T}\right)}+\left\|\frac{1+\varepsilon\left|v^{\varepsilon}\right|^{2}}{1+Q_{L^{1}\left(Q_{T}\right)}}\right\| v_{L^{2}\left(Q_{T}\right)}\left\|v_{i}^{\varepsilon}-v_{i}^{*}\right\|_{L^{2}\left(Q_{T}\right)} \\
& \leq\left\|v_{i}^{\varepsilon}\right\|_{L^{2}\left(Q_{T}\right)} \cdot\left\|v_{j}^{\varepsilon}-v_{j}^{*}\right\|_{L^{2}\left(Q_{T}\right)}+\left\|v_{j}^{*}\right\|_{L^{2}} \\
& \quad+\left\|\frac{\varepsilon\left|v^{\varepsilon}\right|^{2} v_{i}^{*} v_{j}^{*}}{1+\varepsilon\left|v^{\varepsilon}\right|^{2}}\right\|_{L^{1}\left(Q_{T}\right)} .
\end{aligned}
$$

Under the conditions of the lemma, $\left\|v^{\varepsilon}-v^{*}\right\|_{L^{2}\left(Q_{T}\right)} \rightarrow 0$ as $\varepsilon \rightarrow 0$ and $\left\|v^{\varepsilon}\right\|_{L^{2}\left(Q_{T}\right)},\left\|v^{*}\right\|_{L^{2}\left(Q_{T}\right)}$ are uniformly bounded. Hence, to complete the proof it is sufficient to show that the third term in (4.16) also tends to zero as $\varepsilon \rightarrow 0$.

The convergence $v^{\varepsilon} \rightarrow v^{*}$ a.e. on $Q_{T}$ implies that $\frac{\varepsilon\left|v^{\varepsilon}\right|^{2} v_{i}^{*} v_{j}^{*}}{1+\varepsilon\left|v^{\varepsilon}\right|^{2}} \rightarrow 0$ a.e. on $Q_{T}$ as $\varepsilon \rightarrow 0$. Furthermore, $\left|\frac{\varepsilon\left|v^{\varepsilon}\right|^{2} v_{i}^{*} v_{j}^{*}}{1+\varepsilon\left|v^{\varepsilon}\right|^{2}}\right| \leq\left|v_{i}^{*} v_{j}^{*}\right|$ and $v_{i}^{*} v_{j}^{*} \in L^{1}\left(Q_{T}\right)$. Thus, $\left\|\frac{\varepsilon\left|v^{\varepsilon}\right|^{2} v_{i}^{*} v_{j}^{*}}{1+\varepsilon\left|v^{\varepsilon}\right|^{2}}\right\|_{L^{1}\left(Q_{T}\right)} \rightarrow 0$ as $\varepsilon \rightarrow 0$ by the Lebesgue theorem $[6$, Theorem $1.1]$.

We are now in the position to prove the weak solvability of the initialboundary value problem for (4.13).

Theorem 4.5. Let $n \leq 4$ and the condition (4.14) be fulfilled. Let also the matrix-functions $b(i, j, \cdot)$ and the vector-functions $c(i, j, \cdot)$ be essentially bounded for $i, j=\overline{1, n}$. Then for every $f \in L^{2}((0, T), H)$ and $v^{0} \in H$ there exists at least one weak solution

$$
v \in L^{2}((0, T), V) \text { with } v^{\prime} \in L^{1}\left((0, T), V^{*}\right)
$$


of the problem (4.13), (1.2)-(1.4) which satisfies the inequalities

$$
\begin{gathered}
\max _{t \in[0, T]}\|v(t)\|_{H}+\sum_{i=1}^{n}\left\|\frac{\partial v}{\partial x_{i}}\right\|_{L^{2}((0, T), H)} \leq C\left(1+\|f\|_{L^{2}((0, T), H)}+\left\|v^{0}\right\|_{H}\right), \\
\left\|v^{\prime}\right\|_{L^{1}\left((0, T), V^{*}\right)} \leq C\left(1+\|f\|_{L^{2}((0, T), H)}+\left\|v^{0}\right\|_{H}\right)^{2}
\end{gathered}
$$

with $C$ independent of $v, f$ and $v^{0}$.

Proof. It is easy to see that in the case of equation (4.13) conditions $\left.A_{1}\right)-$ $\left.A_{3}\right)$ are fulfilled. The terms $B_{1}(v)$ and $C(v)$ are absent. Hence, conditions $\left.M_{1}\right)-M_{2}$ ) are also fulfilled. Thus, by theorem 3.2, every approximating equation

$$
v^{\prime}+\mu_{0} A v-D_{\varepsilon}(v)-\int_{0}^{t} G(t, s, v(s)) d s=f, \quad \varepsilon>0
$$

has a solution $v \in W$ such that $v(0)=v^{0}$.

Choose an arbitrary sequence of positive numbers $\left\{\varepsilon_{m}\right\}$ converging to zero. Let $\left\{v^{m}\right\}, v^{m} \in W$ be a sequence of solutions of the corresponding operator equations $\left(4.15_{\varepsilon_{m}}\right)$. By estimates (4.1), (4.2), the sequence $\left\{v^{m}\right\}$ is bounded. Hence, repeating the arguments of the proof of theorem 4.3, without loss of generality, we may assume that

$$
\begin{aligned}
& v^{m} \rightarrow v^{*} \text { weakly in } X, \\
& v^{m} \rightarrow v^{*} \quad * \text {-weakly in } L^{\infty}((0, T), H), \\
& v^{m} \rightarrow v^{*} \text { strongly in } L^{2}\left(Q_{T}\right), \\
& v^{m} \rightarrow v^{*} \text { a. e. in } Q_{T}, \\
& \left(v^{m}\right)^{\prime}--\rightarrow\left(v^{*}\right)^{\prime} \quad \text { (in the sense of distributions). }
\end{aligned}
$$

It is known that linear continuous operators are weakly continuous. Hence,

$$
\begin{aligned}
A v^{m} & \rightarrow A v^{*} \text { weakly in } X, \\
\int_{0}^{t} G\left(t, s, v^{m}(s)\right) d s & \rightarrow \int_{0}^{t} G\left(t, s, v^{*}(s)\right) d s \text { weakly in } X^{*} .
\end{aligned}
$$

Therefore [6, Lemma 1.10], they converge in the sense of distributions. Furthermore, in lemma 4.2 we showed that $D_{\varepsilon_{m}}\left(v^{m}\right)--\rightarrow K\left(v^{*}\right)$ in the sense of distributions on $[0, T]$ with values in $\mathcal{V}^{*}$. Thus, passing to the limit in the sense of such distributions in the equality

$$
\left(v^{m}\right)^{\prime}+\mu_{0} A v^{m}-D_{\varepsilon_{m}}\left(v^{m}\right)-\int_{0}^{t} G\left(t, s, v^{m}(s)\right) d s=f,
$$


we obtain the following distributional equality

$$
\left(v^{*}\right)^{\prime}+\mu_{0} A v^{*}-K\left(v^{*}\right)-\int_{0}^{t} G\left(t, s, v^{*}(s)\right) d s=f .
$$

All the terms, but the first one, are contained in $L^{1}\left((0, T), V^{*}\right)$, which implies $\left(v^{*}\right)^{\prime} \in L^{1}\left((0, T), V^{*}\right)$. Therefore, $v^{*}$ is a solution of the equation (4.15). Furthermore, since the sequence $\left\{v^{m}\right\}$ is bounded in the norm of the space $L^{\infty}((0, T), H)$ and $\left\{v^{m}(0)\right\}$ is bounded in $H$, we may assume, without loss of generality, that $v^{m}(0) \rightarrow v^{*}(0)$ weakly in $H$, i.e., $v^{*}(0)=v^{0}$. All a priori estimates for solutions $v^{m}$ are also valid for the function $v^{*}$. Hence, inequalities (4.17) hold.

\section{REFERENCES}

[1] Yu. Ya. Agranovich and P. E. Sobolevskii, Motion of a nonlinear viscoelastic fluid, Dokl. Acad. Nauk SSSR, 314 (1990), 521-525. (Russian). Transl. Soviet Math. Dokl. 42 (1991), 274-278.

[2] Yu. Ya. Agranovich and P. E. Sobolevskii, Investigation of a mathematical model of a viscoelastic fluid, Dokl. Akad. Nauk Ukrain. SSR, Ser. A, No. 10, (1989), 3-6. (Russian)

[3] R. R. Akhmerov, M. I. Kamenskii, A. S. Potapov, A. E. Rod kina and B. N. Sadovskii, Measures of noncompactness and condensing operators, "Nauka" Sibirsk. Otdel., Novosibirsk, 1986, 266 pp. (Russian).

[4] V. T. Dmitrienko and V. G. Zvyagin, Homotopy classification of a class of continuous mappings, Mat. Zametki, 31 (1982), 801-812.

[5] G. Duvaut and J.-L. Lions, Inequalities in mechanics and physics, "Nauka", Moscow, 1980, 384 pp. (Russian, translated from French).

[6] H. Gaevskii, K. Greger and K. Zakharias, Nonlinear operator equations and operator differential equations, "Mir", Moscow, 1978, 336 pp. (Russian, translated from German).

[7] L. V. Kantorovich and G. P. Akilov, Functional analysis, 3rd Edition, "Nauka", Moscow, 1984, 752 pp. (Russian).

[8] N. A. Karazeeva, A. A. Kotsiolis and A. P. Oskolkov, Dynamical systems generated by initial-boundary value problems for equations of motion of linear viscoelastic fluids, Boundary value problems of mathematical physics 14, Trudy Mat. Inst. Steklov, 188 (1990), 59-87. (Russian).

[9] M. A. Krasnoselskii, Topological methods in the theory of nonlinear integral equations, "Gostekhizdat", Moscow, 1956. (Russian).

[10] O. A. Ladyzhenskaya, Mathematical questions of dynamics of viscous incompressible fluid, "Nauka", Moscow, 1970. (Russian).

[11] O. A. Ladyzhenskaya, Some directions in the research carried out at the Laboratory of Mathematical Physics of the Leningrad Branch of the Institute of Mathematics, Theoretical and mathematical physics, Trudy Mat. Inst. Steklov, 175 (1986), 217245. (Russian).

[12] J.-L. Lions, Some methods of solution of nonlinear boundary problems, "Mir", Moscow, 1972. (Russian).

[13] V. G. Litvinov, Motion of a nonlinearly viscous fluid, "Nauka", Moscow, 1982, 375 pp. (Russian).

[14] P. E. Sobolevskii, The existence of solutions of a mathematical model of a nonlinear viscous fluid, Dokl. Akad. Nauk SSSR, 285 (1985), 44-48. (Russian).

[15] R. Temam, Navier-Stokes equations, theory and numerical analysis, "Mir", Moscow, 1981. (Russian). 
[16] M. I. Vishik, A. I. Komech and A. V. Fursikov, Some mathematical problems of statistical hydrodynamics, Uspekhi Mat. Nauk, 34 (1979), 135-210. (Russian).

Mathematics Department

Voronezh State University

Universitetskaya Pl. 1

394693 VORONEZH, RUSSIA

E-mail address: zvyagin@alg.vsu.ru 


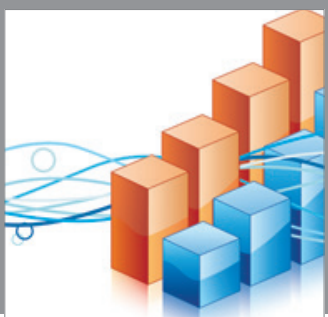

Advances in

Operations Research

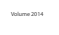

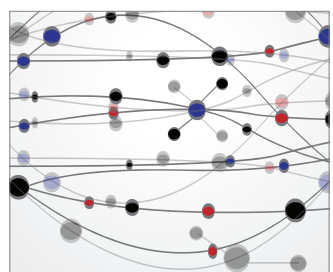

\section{The Scientific} World Journal
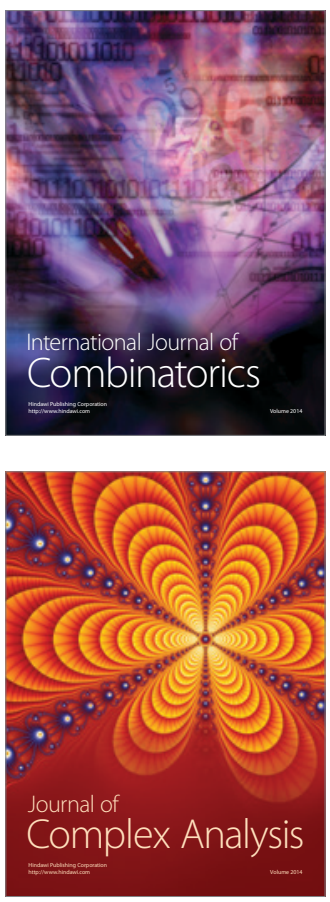

International Journal of

Mathematics and

Mathematical

Sciences
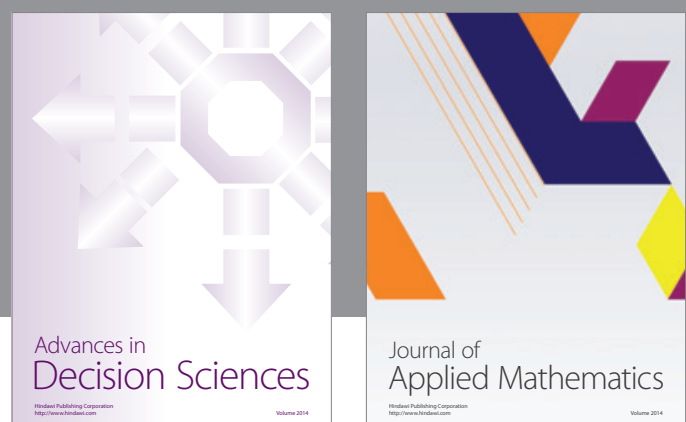

Journal of

Applied Mathematics
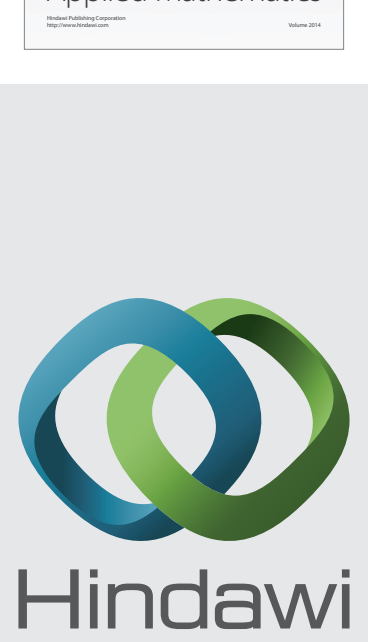

Submit your manuscripts at http://www.hindawi.com
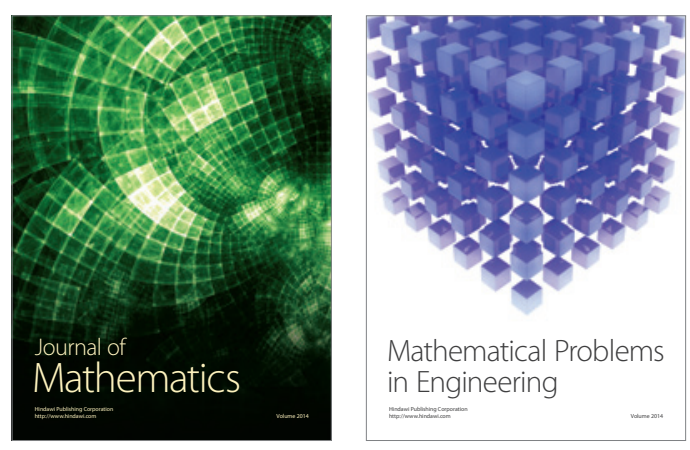

Mathematical Problems in Engineering
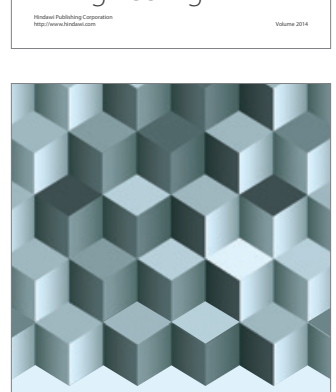

Journal of

Function Spaces
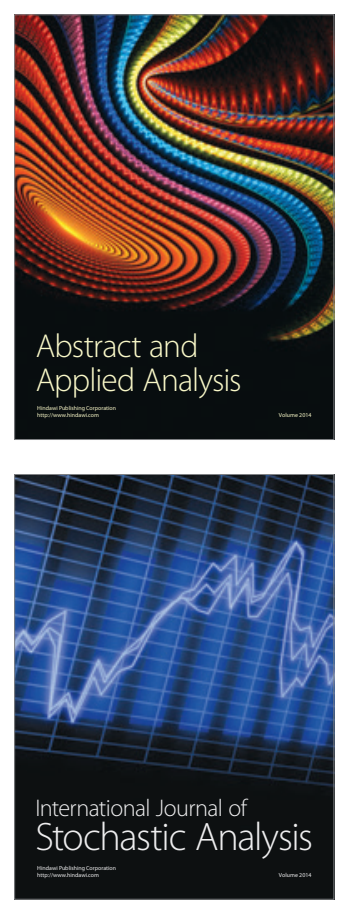

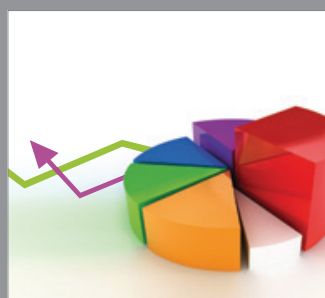

ournal of

Probability and Statistics

Promensencen
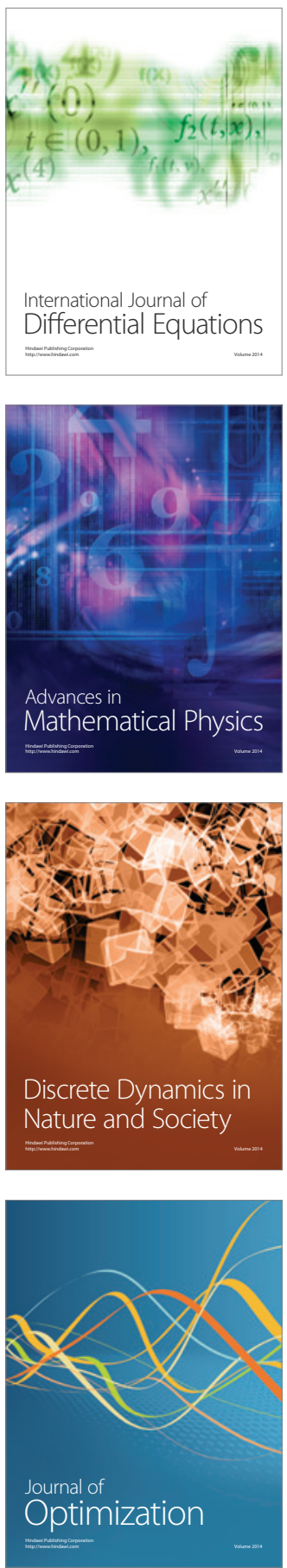